\title{
Active Control Optimization for Minimizing the Dynamic Response of Functionally Graded Plate
}

\author{
M. E. Fares, M. Kh. Elmarghany, and Doaa Atta \\ Department of Mathematics, Faculty of Science, Mansoura University, Mansoura 35516, Egypt \\ Correspondence should be addressed to M. E. Fares; me_fares@yahoo.com and Doaa Atta; doaaatta44@gmail.com
}

Received 10 July 2014; Accepted 22 September 2014; Published 27 October 2014

Academic Editor: Ronald M. Barrett

Copyright ( 2014 M. E. Fares et al. This is an open access article distributed under the Creative Commons Attribution License, which permits unrestricted use, distribution, and reproduction in any medium, provided the original work is properly cited.

\begin{abstract}
A control optimization problem for functionally graded (FG) plates is presented using a first-order shear deformation plate theory including through-the-thickness normal strain effect. The aim of the optimization is to minimize the vibrational response of FG plate with constraints on the control energy used in the damping process. An active control optimization is presented to determine the optimal level of a closed loop control function. Plate thickness and a homogeneity parameter of FG plates are used as design variables. Numerical results for the optimal control force and the total energy for a simply supported FG plate are given. The influence of through-the-thickness normal strain effect on the accuracy of the obtained results is illustrated. The effectiveness of the present control and design procedure is examined.
\end{abstract}

\section{Introduction}

The rapid development in various industrial fields requires new materials that can serve useful functions under certain conditions. In recent years, functionally graded materials (FGMs) have gained considerable attention in many engineering applications, and they are considered as a potential structural material for future high-speed spacecraft and power generation industries. Several studies have been performed to analyze the behavior of functionally graded plates [1].

In the aerospace industry and many other engineering applications, the suppression of excessive vibrations occurring in large structures represents one of the most pressing and difficult problems facing structural designers. An effective means of suppressing excessive vibrations is by active structural control. Thus, there is need for new light materials possessing a high degree of flexibility and with very low natural damping. These factors motivated the development for more accurate tools of analysis and rigorous design methods [2-7]. A series of publications has appeared concerning the vibration control and design optimization of composite structures. Grandhi [8] studied the structural and control optimization of space structures. Sloss et al. [9] presented an integrated approach to solve multiobjective design and control optimization for many composite structures. Adali et al. [10, 11], Sloss et al. [12], Sadek et al. [13], and Fares et al. [14-16] have studied the optimal design and control optimization of composite laminated plates and shells subjected to thermomechanical loadings.

In another work of Fares et al. [17], nonlinear optimization schemes are presented to solve multiobjective design and control problems for composite laminated plates. Other studies on this topic may be found in $[18,19]$.

Many studies indicate that the inclusion of the normal strain and the transverse shear effects are very important for predicting accurate vibrational responses of composite plates and shells, particularly, for those made of FGMs; see, for example, [20]. In the work [20], it is showed that the neglecting of these effects may cause errors in predicting the deflections and frequencies in FG plates reaching $30 \%$. However, most studies related to the topic of design and control optimization of composite plates and shells were carried out based on classical theories which neglect the shear deformation and normal strain effects.

The current work deals with the active control of the dynamic response of a FG orthotropic rectangular plate. The present formulation is based on a consistent first-order 
plate theory including the shear deformation and throughthe-thickness normal strain effects [20]. The design and control objective are to minimize the dynamic response with minimum expenditure of control energy. The total elastic energy is taken as a measure of the dynamic response. The expenditure of the control energy is limited by attaching to the control objective a functional including the control force. The necessary and sufficient condition for optimal stabilization in the Liapunov-Bellman sense [21] is used to determine the optimal control force and controlled deflections. Numerical examples are given to assess the efficiency of the present design control approach in the damping process for FG plate.

\section{Formulation and Basic Equations}

Consider an elastic plate of uniform thickness $h$, length $a$, and width $b$. The plate is composed of an orthotropic elastic material varying in the thickness direction. Let a Cartesian coordinates system $\left(x_{1}, x_{2}, x_{3}\right)$ be taken such that the midplane coincides with the $x_{1} x_{2}$ plane and the plate occupies the following region:

$$
0 \leq x_{1} \leq a, \quad 0 \leq x_{2} \leq b, \quad-\frac{h}{2} \leq x_{3} \leq \frac{h}{2} .
$$

Let the upper surface of the plate $\left(x_{3}=h / 2\right)$ be subjected to a transverse distributed load $q\left(x_{1}, x_{2}, x_{3}\right)$ which may be taken as a control force.

The present formulation is based on a first-order theory including a normal strain and shear deformation effects derived using a mixed variational approach. This theory preserves the transverse shear stresses which vanish on the top and the bottom of the plate surfaces; therefore, the condition of introducing shear correction factors is not required. The displacement field is taken in the form [20]:

$$
\begin{gathered}
u_{1}=u_{01}+\psi_{1} x_{3}, \quad u_{2}=u_{02}+\psi_{2} x_{3}, \\
w=w_{0}+\alpha w_{1} x_{3}+\beta w_{2} x_{3}^{2},
\end{gathered}
$$

where $\left(u_{1}, u_{2}, w\right)$ are the displacements along $x_{1}, x_{2}$, and $x_{3}$ directions, respectively, $\left(u_{01}, u_{02}, w_{0}\right)$ are the displacements of a point on the mid-plane, and $\left(\psi_{1}, \psi_{2}\right)$ are the slopes in the $x_{1} x_{3}$ and $x_{2} x_{3}$ planes due to bending only (slope rotations). The constants $\alpha$ and $\beta$ may take the values 0 or 1 to study the effect of the normal strain on the control process.

The infinitesimal strains $\varepsilon_{i j}$ associated with displacements (2) are given by

$$
\begin{gathered}
\varepsilon_{i i}=u_{0 i, i}+\psi_{i, i} x_{3}, \\
\varepsilon_{i 3}=\psi_{i}+w_{0, i}+\alpha w_{1, i} x_{3}+\beta w_{2, i} x_{3}^{2}, \\
(i=1,2),
\end{gathered}
$$

$$
\begin{gathered}
\varepsilon_{33}=\alpha w_{1}+2 \beta w_{2} x_{3} \\
\varepsilon_{12}=\left(u_{01,2}+u_{02,1}\right)+\left(\psi_{1,2}+\psi_{2,1}\right) x_{3}
\end{gathered}
$$

where the suffix $(, i)$ denotes partial differentiation with respect to the coordinate $x_{i}$.

Moreover, the stresses are taken in the following form:

$$
\begin{gathered}
\sigma_{i j}=\frac{N_{i j}}{h}+\frac{12 M_{i j}}{h^{3}} x_{3}, \\
\sigma_{i 3}=\frac{3 Q_{i 3}}{2 h}\left[1-\left(\frac{x_{3}}{h / 2}\right)^{2}\right], \\
\sigma_{33}=\left(\frac{3 N_{33}}{2 h}+\frac{30 M_{33}}{h^{3}} x_{3}\right)\left[1-\left(\frac{x_{3}}{h / 2}\right)^{2}\right] \\
+\frac{q_{1}}{4}\left[\left(\frac{x_{3}}{h / 2}\right)^{2}-\left(\frac{x_{3}}{h / 2}\right)-2\right]\left(1+\frac{x_{3}}{h / 2}\right) \\
+\frac{q_{2}}{4}\left[\left(\frac{x_{3}}{h / 2}\right)^{2}+\left(\frac{x_{3}}{h / 2}\right)-2\right]\left(1-\frac{x_{3}}{h / 2}\right) .
\end{gathered}
$$

The governing equations of this system are determined using the dynamic modified version of the mixed variational principle of Ressiner [20]:

$$
\begin{gathered}
\delta u_{0 i}: N_{i i, i}+N_{i j, j}=I_{1} \ddot{u}_{0 i}+I_{2} \ddot{\psi}_{i}, \\
\delta \psi_{i}: M_{i i, i}+M_{i j, j}-Q_{i 3}=I_{2} \ddot{u}_{0 i}+I_{3} \ddot{\psi}_{i}, \\
(i, j=1,2, \quad i \neq j), \\
\delta w_{0}: Q_{13,1}+Q_{23,2}+q=I_{1} \ddot{w}_{0}+\alpha I_{2} \ddot{w}_{1}+\beta I_{3} \ddot{w}_{2}, \\
\delta w_{1}: h q-N_{33}=I_{2} \ddot{w}_{0}+\alpha I_{3} \ddot{w}_{1}+\beta I_{4} \ddot{w}_{2}, \\
\delta w_{2}: \frac{h^{2}\left(Q_{13,1}+Q_{23,2}\right)}{20}-2 M_{33}+\frac{9 h^{2} q}{20} \\
=I_{3} \ddot{w}_{0}+\alpha I_{4} \ddot{w}_{1}+\beta I_{5} \ddot{w}_{2} .
\end{gathered}
$$


Moreover, the constitutive equations are

$$
\begin{gathered}
\left\{\begin{array}{l}
N_{11} \\
N_{22} \\
N_{33} \\
N_{12} \\
M_{11} \\
M_{22} \\
M_{33} \\
M_{12}
\end{array}\right\}=\left[\begin{array}{cccccccc}
A_{11} & A_{12} & A_{13} & 0 & B_{11} & B_{12} & B_{13} & 0 \\
A_{12} & A_{22} & A_{23} & 0 & B_{12} & B_{22} & B_{23} & 0 \\
A_{13} & A_{23} & A_{33} & 0 & \bar{B}_{13} & \bar{B}_{23} & B_{33} & 0 \\
0 & 0 & 0 & A_{66} & 0 & 0 & 0 & B_{66} \\
B_{11} & B_{12} & \bar{B}_{13} & 0 & D_{11} & D_{12} & D_{13} & 0 \\
B_{12} & B_{22} & \bar{B}_{23} & 0 & D_{12} & D_{22} & D_{23} & 0 \\
B_{13} & B_{23} & B_{33} & 0 & D_{13} & D_{23} & D_{33} & 0 \\
0 & 0 & 0 & B_{66} & 0 & 0 & 0 & D_{66}
\end{array}\right]^{-1}\left[\begin{array}{c}
u_{01,1}-F_{13} \\
u_{02,2}-F_{23} \\
\alpha w_{1}-F_{33} \\
u_{01,2}+u_{02,1} \\
\psi_{1,1}-K_{13} \\
\psi_{2,2}-K_{23} \\
2 \beta w_{2}-K_{33} \\
\psi_{1,2}+\psi_{2,1}
\end{array}\right] \\
Q_{i 3}=\frac{1}{A_{k k}}\left[\begin{array}{l}
\psi_{i}+w_{0, i}+\left(\frac{\beta h^{2} w_{2, i}}{20}\right)+H_{i}
\end{array}\right], \quad\left(i=1,2 ; k=i+\left(\frac{4}{i}\right)\right) .
\end{gathered}
$$

The stress and moment resultants $N_{i j}, M_{i j}$, and $Q_{i j}$ in (5) and (6a) and (6b) can be expressed as

$$
\begin{gathered}
\left(N_{i j}, M_{i j}\right)=\int_{-h / 2}^{h / 2}\left(1, x_{3}\right) \sigma_{i j} d x_{3}, \quad(i, j=1,2,3) \\
\left(Q_{k 3}\right)=\int_{-h / 2}^{h / 2} \sigma_{k 3} d x_{3}, \quad(k=1,2),
\end{gathered}
$$

and the stiffness coefficients $A_{i j}, B_{i j}$, and $D_{i j}$ are given in Appendix A.

In the present control problem, all edges of the plate are taken simply supported (SSSS), and then, the following conditions must be satisfied at the plate edges:

$$
\begin{array}{ll}
u_{01}=w_{0}=w_{1}=w_{2}=\psi_{2}=N_{11}=M_{11}=0, & x_{1}=0, a \\
u_{02}=w_{0}=w_{1}=w_{2}=\psi_{1}=N_{22}=M_{22}=0, & x_{2}=0, b .
\end{array}
$$

\section{The Optimal Design and Control Problem}

The objective of the present control problem is to minimize the dynamic response of the plate in a specified time $0 \leq$ $t \leq \tau \leq \infty$ with the minimum possible expenditure of force $q\left(x_{1}, x_{2}, t\right)$, when the plate is subjected to the following initial disturbances:

$$
\begin{aligned}
& w\left(x_{1}, x_{2}, 0\right)=\bar{A}\left(x_{1}, x_{2}\right), \\
& \dot{w}\left(x_{1}, x_{2}, 0\right)=\bar{B}\left(x_{1}, x_{2}\right) .
\end{aligned}
$$

The dynamic response of the plate is measured by a cost functional including the energy of the plate which is a function of displacements, its derivatives, and the velocity. The control force $q\left(x_{1}, x_{2}, t\right)$ is introduced into the formulation by taking a performance index which comprises a weighted sum of the energy (cost functional) and penalty functional involving the control energy. Then, the mathematical formulation of the control problem can be reduced to determine the optimization variable $q$ that minimizes the following control objective:

$$
J=\mu_{1} J_{1}+\mu_{2} J_{2}+\mu_{3} J_{3}
$$

$$
\begin{array}{r}
J_{1}=\frac{1}{2} \int_{0}^{\infty} \int_{0}^{b} \int_{0}^{a} \int_{-h / 2}^{h / 2} \varepsilon_{i j} \sigma_{i j} d x_{3} d x_{1} d x_{2} d t \\
(i, j=1,2,3),
\end{array}
$$

$$
\begin{gathered}
J_{2}=\frac{1}{2} \int_{0}^{\infty} \int_{0}^{b} \int_{0}^{a} \int_{-h / 2}^{h / 2} \rho\left(\dot{u}_{1}^{2}+\dot{u}_{2}^{2}+\dot{w}^{2}\right) d x_{3} d x_{1} d x_{2} d t \\
J_{3}=\int_{0}^{\infty} \int_{0}^{b} \int_{0}^{a} q^{2}\left(x_{1}, x_{2}, t\right) d x_{1} d x_{2} d t
\end{gathered}
$$

where $\mu_{1}, \mu_{2}$, and $\mu_{3}$ are positive constant weighting factors and $J_{1}$ and $J_{2}$ represent the strain and the kinetic energies of the plate, respectively. The functional $J_{3}$ is a penalty term involving the control function $q \in L^{2}$, where $L^{2}$ denotes the set of all bounded square integrable functions on the domain of solution $\left\{0 \leq x_{1} \leq a, 0 \leq x_{2} \leq b, 0 \leq t \leq \tau \leq \infty\right\}$. Note that, the functional $J$ is a quadratic and, hence, differentiable and positive definite on the domain of solution.

It is obvious that the solution of the present optimal control problem requires finding a solution of the initial boundary-value problem (5), (6a), (6b), (8), and (9) that minimizes the functional (10). Moreover, the design optimization procedure aims to minimize the actively controlled dynamic responses using geometry and material parameters as design variables. 


\section{Solution Procedure}

The displacement functions $\left(u_{01}, u_{02}, w_{0}, \psi_{1}, \psi_{2}, w_{1}, w_{2}\right)$ and the mechanical load $q$ may be taken for simply supported plate in the form:

$$
\begin{gathered}
\left(u_{0 i}, \psi_{i}\right)=\sum_{m, n}\left(U_{i m n}(t), \Psi_{i m n}(t)\right) X_{i, i} X_{i+1}, \\
(i=1 \longrightarrow i+1=2, i=2 \longrightarrow i+1=1) \\
\left(w_{j}, q\right)=\sum_{m, n}\left(W_{j m n}(t), q_{m n}(t)\right) X_{1} X_{2}, \quad(j=0,1,2) \\
X_{1}\left(x_{1}\right)=\sin \mu_{m} x_{1}, \quad \mu_{m}=\frac{m \pi}{a}, \\
X_{2}\left(x_{2}\right)=\sin \lambda_{n} x_{2}, \quad \lambda_{n}=\frac{n \pi}{b},
\end{gathered}
$$

where $U_{i m n}(t), \Psi_{i m n}(t), W_{j m n}(t)$, and $q_{m n}(t)$ are unknown functions of the time $t$.

Using (6a) and (6b) we can get the governing equations (5) in terms of the displacements $\left(u_{01}, u_{02}, w_{0}, \psi_{1}, \psi_{2}, w_{1}, w_{2}\right)$. For these equations, the in-plane inertia terms are neglected. Substituting expressions (11) into the resulting equations, we get the following time equations:

$$
\begin{aligned}
& U_{1 i} U_{1 m n}+U_{2 i} U_{2 m n}+\Psi_{1 i} \Psi_{1 m n}+\Psi_{2 i} \Psi_{21 m n} \\
& \quad+W_{0 i} W_{0 m n}+W_{1 i} W_{1 m n}+W_{2 i} W_{2 m n} \\
& =\ddot{W}_{0 m n} \bar{W}_{i}\left(\delta_{i 3}+\delta_{i 6}+\delta_{i 7}\right)+Q_{i} q_{m n}, \quad(i=1,2, \ldots, 7),
\end{aligned}
$$

where $\delta_{i j}$ is the kronecker delta and the coefficients $U_{1 i}, U_{2 i}$, $\Psi_{1 i}, \Psi_{2 i}, W_{0 i}, W_{1 i}, W_{2 i}, Q_{i}$ are given in Appendix B. Solving the system of (12), one gets an equation of the time-dependent functions $q_{m n}$ and $W_{0 m n}$ only,

$$
\ddot{W}_{0 m n}+\omega^{2} W_{0 m n}=l q_{0}, \quad \omega^{2}=\frac{\Delta_{1}}{\Delta_{3}}, l=\frac{\Delta_{2}}{\Delta_{3}},
$$

where $\Delta_{1}, \Delta_{2}$, and $\Delta_{3}$ are given in Appendix B.

Again, using (2), (3), (4), and (11) with the objective functional (10), we get

$$
\begin{aligned}
J= & \sum_{m n} \int_{0}^{\infty} \bar{J}_{m n} d t \\
\bar{J}_{m n}= & K_{1} W_{0 m n}^{2}+K_{2} W_{0 m n} q_{m n}+K_{3} q_{m n}^{2} \\
& +K_{4} \dot{W}_{0 m n}^{2}+K_{5} \dot{W}_{0 m n} \dot{q}_{m n}+K_{6} \dot{q}_{m n}^{2},
\end{aligned}
$$

where the coefficients $K_{i}(i=1,2, \ldots, 6)$ are given in Appendix B.
Now the optimal control problem is to find the control function $q_{m n}^{\mathrm{opt}}(t)$ that minimizes the functional (14); that is,

$$
J\left(q_{m n}^{\mathrm{opt}}\right) \leq J\left(q_{m n}\right), \quad \forall q_{m n}(t) \in L^{2}([0, \infty))
$$

that is,

$$
\min _{q_{m n}} J=\min \sum J_{m n}=\sum \min _{q_{m n} \in L^{2}} J
$$

The minimization of the dynamic response using the control force $q_{0}$ can be carried out using the Liapunov-Bellman method [21] which is considered an effective approach for the solution. The necessary and sufficient condition for minimization of the functional (14) in the Liapunov-Bellman sense is

$$
\min _{q}\left[\frac{\partial \bar{V}}{\partial W_{0 m n}} \dot{W}_{0 m n}+\frac{\partial \bar{V}}{\partial \dot{W}_{0 m n}} \ddot{W}_{0 m n}+\bar{J}\right]=0 ;
$$

provided that the Liapunov function $\bar{V}$ is a positive definite, then

$$
\begin{array}{r}
\bar{V}=A W_{0 m n}^{2}+2 B W_{0 m n} \dot{W}_{0 m n}+C \dot{W}_{0 m n}^{2}, \\
A>0, \quad C>0, \quad A C>B^{2} .
\end{array}
$$

Substituting expressions (13) and (14) into (17) one gets

$$
\begin{gathered}
\min _{q}\left[\frac{\partial \bar{V}}{\partial W_{0 m n}} \dot{W}_{0 m n}+\frac{\partial \bar{V}}{\partial \dot{W}_{0 m n}}\left(l q_{m n}-\omega^{2} W_{0 m n}\right)\right. \\
+K_{1} W_{0 m n}^{2}+K_{2} W_{0 m n} q_{m n}+K_{3} q_{m n}^{2} \\
\left.+K_{4} \dot{W}_{0 m n}^{2}+K_{5} \dot{W}_{0 m n} \dot{q}_{m n}+K_{6} \dot{q}_{m n}^{2}\right]=0 .
\end{gathered}
$$

This condition gives that

$$
l \frac{\partial \bar{V}}{\partial \dot{W}_{0 m n}}+K_{2} W_{0 m n}+2 K_{3} q_{m n}^{\mathrm{opt}}=0 .
$$

Substituting the Liapunov function (18) into the previous expression, we can get the minimum control force $q_{m n}^{\text {opt }}$ in the form:

$$
q_{m n}^{\mathrm{opt}}=\frac{-1}{2 K_{3}}\left(2 B l+K_{2}\right) W_{0 m n}-\frac{1}{K_{3}} C l \dot{W}_{0 m n} .
$$

Again, using (18) with the expression (19), we obtain

$$
\begin{aligned}
\min _{q}[( & \left.2 A W_{0 m n} \dot{W}_{0 m n}+2 B \dot{W}_{0 m n}^{2}\right) \\
& +\left(2 B W_{0 m n}+2 C \dot{W}_{0 m n}\right)\left(l q_{m n}-\omega^{2} W_{0 m n}\right) \\
& +K_{1} W_{0 m n}^{2}+K_{2} W_{0 m n} q_{m n}+K_{3} q_{m n}^{2} \\
& \left.+K_{4} \dot{W}_{0 m n}^{2}+K_{5} \dot{W}_{0 m n} \dot{q}_{m n}+K_{6} \dot{q}_{m n}^{2}\right]=0 .
\end{aligned}
$$


Using (21) with the last expression and equating the coefficients of $\dot{W}_{0 m n}^{2}, W_{0 m n}^{2}$, and $W_{0 m n} \dot{W}_{0 m n}$ by zeroes, the following system of algebraic equations is obtained:

$$
\begin{gathered}
C^{2}\left(a_{1} B^{2}+a_{2} B+a_{3}\right)+a_{4} B^{2}+a_{5} B+a_{6}=0, \\
C^{2}\left(a_{7} C^{2}+a_{8} B+a_{9}\right)+a_{10} B^{2}+a_{11} B+a_{12}=0, \\
a_{13} A+C\left(a_{14} C^{2}+a_{15} B C^{2}+a_{16} B^{2}+a_{17} B+a_{18}\right)=0,
\end{gathered}
$$

where the coefficients $a_{i}(i=1,2, \ldots, 18)$ are given in Appendix C.

Using (14) and (21), we obtain the following differential equation:

$$
\begin{gathered}
\ddot{W}_{0 m n}+\alpha_{m n} \dot{W}_{0 m n}+\Omega_{m n}^{2} W_{0 m n}=0, \\
\alpha_{m n}=-l c_{2}, \quad \Omega_{m n}^{2}=\left(\omega^{2}-l c_{1}\right), \\
c_{1}=-\frac{\left(2 B l+K_{2}\right)}{\left(2 K_{3}\right)}, \quad c_{2}=-\frac{C l}{K_{3}} .
\end{gathered}
$$

The solution of (24) for which $2 \Omega_{m n}^{2}>\alpha_{m n}$ is given by

$$
\begin{array}{r}
W_{0 m n}^{\mathrm{opt}}=e^{-\alpha_{m n} t / 2}\left[\beta_{m n} \cos \left(v_{m n} t\right)+\gamma_{m n} \sin \left(v_{m n} t\right)\right], \\
v_{m n}=\Omega_{m n}^{2}-\frac{\alpha_{m n}}{4},
\end{array}
$$

where $\beta_{m n}$ and $\gamma_{m n}$ are unknown coefficients which may be obtained from conditions (9) as

$$
\begin{gathered}
\gamma_{m n}=\frac{\left(\alpha_{m n} \beta_{m n}+2 \bar{A}_{m n}\right)}{\left(2 v_{m n}\right)}, \\
\left(\beta_{m n}, \bar{A}_{m n}\right)=\frac{4}{a b \omega^{2}} \int_{0}^{b} \int_{0}^{a}(\bar{A}, \bar{B}) X_{1} X_{2} d x_{1} d x_{2} .
\end{gathered}
$$

Using (14), (21), and (25), we can get the controlled displacement, the total energy, and the optimal control force. Then we complete the minimization process by determining the optimal design of the plate using geometric and material parameters as design variables.

\section{Homogenization of Material Properties and the Design Objective}

Consider that a FG plate is made of two randomly distributed isotropic constituents (ceramic and metal) and the macroscopic response of the composite is isotropic. The matrix phase, denoted by the subscript 2 , is reinforced by spherical particles as a particulate phase, denoted by the subscript 1 . The homogeneity of this composition is taken to vary only in the thickness direction. The effective elastic moduli of the graded composite may be evaluated from the volume fraction of the constituents $V_{1}$ and $V_{2}$. The effective mass density $\rho$ at a point is given by

$$
\begin{gathered}
\rho=\rho_{1} V_{1}+\rho_{2} V_{2}, \\
V_{1}=V_{1}^{-}+\left(V_{1}^{+}-V_{1}^{-}\right)\left(\frac{1}{2}+\frac{x_{3}}{h}\right)^{p},
\end{gathered}
$$

where $V_{1}^{+}$and $V_{1}^{-}$are, respectively, the volume fractions of the particulate ceramic phase on the top and the bottom surfaces of the plate. The parameter $p$ indicates the volume fraction profile through the thickness. In this case, the elastic coefficients $C_{i j}$ become

$$
\begin{gathered}
C_{11}=C_{22}=C_{33}=\frac{1}{E}, \\
C_{12}=C_{13}=C_{23}=-\frac{v}{E}, \\
C_{44}=C_{55}=C_{66}=\frac{1}{G}, \\
K=\frac{E}{3(1-2 \nu)}, \quad G=\frac{E}{2(1+v)},
\end{gathered}
$$

where $E$ is Young's modulus, $v$ is Poisson's ratio, $G$ is shear modulus, and $K$ is the effective bulk modulus.

The Mori and Tanaka [22] scheme is used to estimate the effective moduli for the regions of the graded microstructure which have a well-defined continuous matrix and a discontinuous particulate phase. The locally effective bulk modulus $K$ and the effective shear modulus $G$ of the composite are given by

$$
\begin{gathered}
\frac{K-k_{1}}{k_{2}-k_{1}}=\frac{V_{2}}{1+\left(1-V_{2}\right)\left(\left(k_{2}-k_{1}\right) /\left(k_{1}+(4 / 3) G_{1}\right)\right)}, \\
\frac{G-G_{1}}{G_{2}-G_{1}}=\frac{V_{2}}{1+\left(1-V_{2}\right)\left(\left(G_{2}-G_{1}\right) /\left(G_{1}+f_{1}\right)\right)}, \\
f_{1}=\frac{G_{1}\left(9 k_{1}+8 G_{1}\right)}{\left(6 k_{1}+12 G_{1}\right)} .
\end{gathered}
$$

The design procedure aims to determine the values of the design variable $r(=a / h)$ and the index $p$ that minimize the energy $J$; that is,

$$
\widehat{J}\left(q_{\mathrm{opt}}, r_{\mathrm{opt}}, p_{\mathrm{opt}}\right)=\min _{r, p} J\left(q_{\mathrm{opt}}, r, p\right)
$$

where $q_{\text {opt }}$ is the optimal control force obtained from the previous control procedure.

\section{Numerical Examples and Discussion}

Numerical results are presented for the total energy $J$ and the control force $q$ for simply supported plates. Let the constituent materials of the FG plate be aluminum (Al) as 
TABLE 1: Comparison of $q_{\text {opt }}, \widetilde{q}_{\text {opt }}, J$, and $\widetilde{J}$ of simply supported square $\mathrm{Al} / \mathrm{ZrO}_{2}$ FG plates with $m=n=1$.

\begin{tabular}{|c|c|c|c|c|c|c|c|}
\hline$p$ & $r$ & $\bar{q}_{\text {opt }}$ & $\tilde{q}_{\text {opt }}$ & $J$ & $\widetilde{J}$ & $q^{*} \%$ & $J^{*} \%$ \\
\hline \multirow{4}{*}{1} & 5 & 0.0312 & 0.0295 & 0.1391 & 0.1401 & 5.5 & 0.7 \\
\hline & 10 & 3.1358 & 3.0909 & 8.1959 & 8.2149 & 1.4 & 0.2 \\
\hline & 15 & 43.401 & 43.133 & 103.64 & 103.79 & 0.61 & 0.1 \\
\hline & 20 & 247.45 & 246.62 & 662.64 & 663.59 & 0.33 & 0.1 \\
\hline \multirow{4}{*}{4} & 5 & 0.0344 & 0.0286 & 0.1062 & 0.1072 & 16.8 & 0.9 \\
\hline & 10 & 3.0272 & 2.8914 & 6.1625 & 6.1720 & 4.5 & 0.2 \\
\hline & 15 & 39.992 & 39.259 & 78.319 & 78.362 & 1.8 & 0.05 \\
\hline & 20 & 221.95 & 219.87 & 499.76 & 500.30 & 0.9 & 0.1 \\
\hline \multirow{4}{*}{10} & 5 & 0.0367 & 0.0284 & 0.0948 & 0.09580 & 22.6 & 1.0 \\
\hline & 10 & 3.0335 & 2.8507 & 5.5484 & 5.5533 & 6.0 & 0.1 \\
\hline & 15 & 39.201 & 38.246 & 70.938 & 70.456 & 2.4 & 0.01 \\
\hline & 20 & 214.70 & 212.04 & 452.59 & 453.06 & 1.2 & 0.1 \\
\hline
\end{tabular}

TABLE 2: Comparison of $q_{\text {opt }}, \widetilde{q}_{\text {opt }}, J$, and $\widetilde{J}$ of simply supported $\mathrm{Al} / \mathrm{ZrO}_{2}$ FG plates with $m=n=1, a / h=5$.

\begin{tabular}{|c|c|c|c|c|c|c|c|}
\hline$p$ & $a / b$ & $\bar{q}_{\mathrm{opt}}$ & $\tilde{q}_{\text {opt }}$ & $J$ & $\widetilde{J}$ & $q^{*} \%$ & $J^{*} \%$ \\
\hline \multirow{3}{*}{1} & 1 & 0.0312 & 0.0295 & 0.1391 & 0.1401 & 5.5 & 0.7 \\
\hline & 2 & 0.062 & 0.059 & 0.014 & 0.014 & 4.5 & 1.33 \\
\hline & 3 & 0.021 & 0.020 & 0.003 & 0.003 & 4.8 & 1.73 \\
\hline \multirow{3}{*}{4} & 1 & 0.344 & 0.286 & 0.106 & 0.107 & 16.8 & 0.94 \\
\hline & 2 & 0.069 & 0.060 & 0.011 & 0.011 & 11.9 & 2.64 \\
\hline & 3 & 0.023 & 0.021 & 0.003 & 0.003 & 10.1 & 2.28 \\
\hline \multirow{3}{*}{10} & 1 & 0.367 & 0.284 & 0.095 & 0.096 & 22.7 & 1.06 \\
\hline & 2 & 0.071 & 0.059 & 0.009 & 0.009 & 15.9 & 2.85 \\
\hline & 3 & 0.024 & 0.021 & 0.002 & 0.002 & 11.7 & 2.84 \\
\hline
\end{tabular}

TABLE 3: Comparison of $q_{\text {opt }}, \widetilde{q}_{\text {opt }}, J$, and $\widetilde{J}$ of simply supported square $\mathrm{Al} / \mathrm{ZrO}_{2}$ FG plates with $m=2, n=1$.

\begin{tabular}{|c|c|c|c|c|c|c|c|}
\hline$p$ & $a / h$ & $\bar{q}_{\mathrm{opt}}$ & $\tilde{q}_{\mathrm{opt}}$ & $J$ & $\widetilde{J}$ & $q^{*} \%$ & $J^{*} \%$ \\
\hline \multirow{3}{*}{1} & 5 & 0.0762 & 0.0599 & 0.0279 & 0.0282 & 21.5 & 1.3 \\
\hline & 10 & 5.9923 & 5.6012 & 1.3429 & 1.3489 & 6.5 & 0.5 \\
\hline & 15 & 88.508 & 85.897 & 14.884 & 14.912 & 3.0 & 0.2 \\
\hline \multirow{3}{*}{4} & 5 & 0.1399 & 0.0603 & 0.0220 & 0.0223 & 56.9 & 1.7 \\
\hline & 10 & 6.5968 & 5.3641 & 1.0158 & 1.0205 & 18.7 & 0.5 \\
\hline & 15 & 81.835 & 80.583 & 11.203 & 11.201 & 8.5 & 0.02 \\
\hline \multirow{3}{*}{10} & 5 & 0.2549 & 0.0597 & 0.0196 & 0.0199 & 76.6 & 1.7 \\
\hline & 10 & 7.0887 & 5.3134 & 0.9099 & 0.9134 & 25.0 & 0.4 \\
\hline & 15 & 89.668 & 79.529 & 10.088 & 10.070 & 11.3 & 0.2 \\
\hline
\end{tabular}

a matrix phase and zirconia as particulate phase with material and geometrical properties given by

$$
\begin{gathered}
\mathrm{ZrO}_{2}: E_{1}=200 \mathrm{GPa}, \quad \nu_{1}=0.3, \\
\rho_{1}=5700 \mathrm{~kg} / \mathrm{m}^{3}, \quad \mathrm{Al}: E_{2}=70 \mathrm{GPa}, \\
\nu_{2}=0.3, \quad \rho_{2}=2702 \mathrm{~kg} / \mathrm{m}^{3}, \\
a=b=0.5 \mathrm{~m}, \quad h=0.02 \mathrm{~m}, \\
\mu_{1}=\mu_{2}=\mu_{4}=1, \quad \mu_{3}=0.1, \\
\bar{A}\left(x_{1}, x_{2}\right)=0.2 X_{1}\left(x_{1}\right) X_{2}\left(x_{2}\right), \quad \bar{B}\left(x_{1}, x_{2}\right)=0, \\
\bar{q}_{\mathrm{opt}}=\frac{a^{3} q_{\mathrm{opt}}}{\left(E_{2} h^{3}\right)}, \quad T=\left(\frac{h t}{a^{2}}\right) \sqrt{\frac{E_{2}}{\rho_{2}}}
\end{gathered}
$$

Tables 1 and 2 contain numerical results for the controlled total energy $J$ and the control force $q_{\text {opt }}$ of simply supported $\mathrm{Al} / \mathrm{ZrO}_{2}$ FG plate with their counterparts $\widetilde{J}$ and $\tilde{q}_{\text {opt }}$ which are calculated neglecting the effect of the normal strains $(\alpha=$ $\beta=0$ ). To study the influence of the normal strain effect on the control process, the following relative errors for the control force $q$ and the total energy $J$ are presented in Tables 1,2 , and 3 for various values of the ratios $r, a / b$ and the index p. In these tables,

$$
q^{*}=\left[\frac{\left(q_{\mathrm{opt}}-\tilde{q}_{\mathrm{opt}}\right)}{q_{\mathrm{opt}}}\right], \quad J^{*}=\left[\frac{(J-\widetilde{J})}{J}\right] .
$$

Note that the relative errors in the control force decrease with the increase of ratio $r$, and these errors increase with increasing the index $p$ of the volume fraction profile, so that 


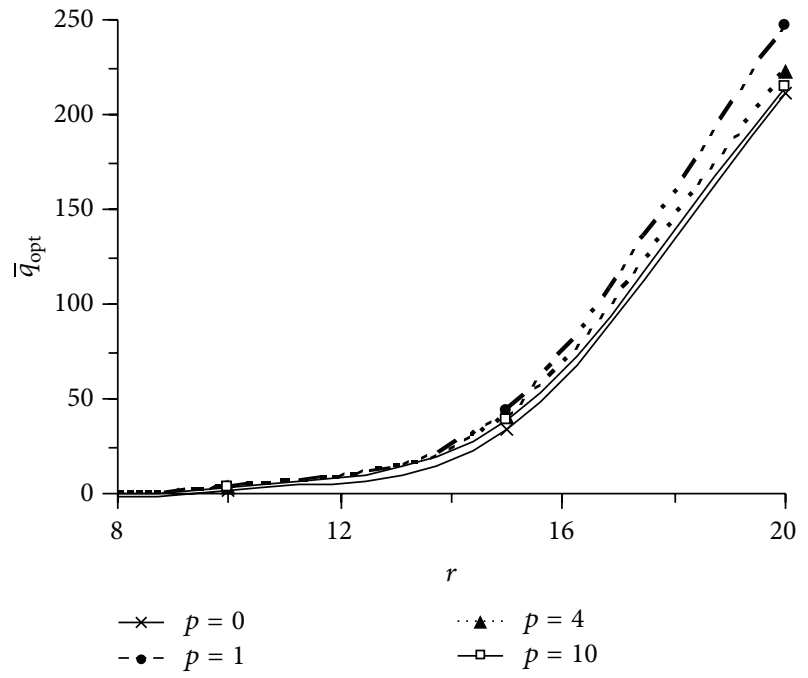

(a)

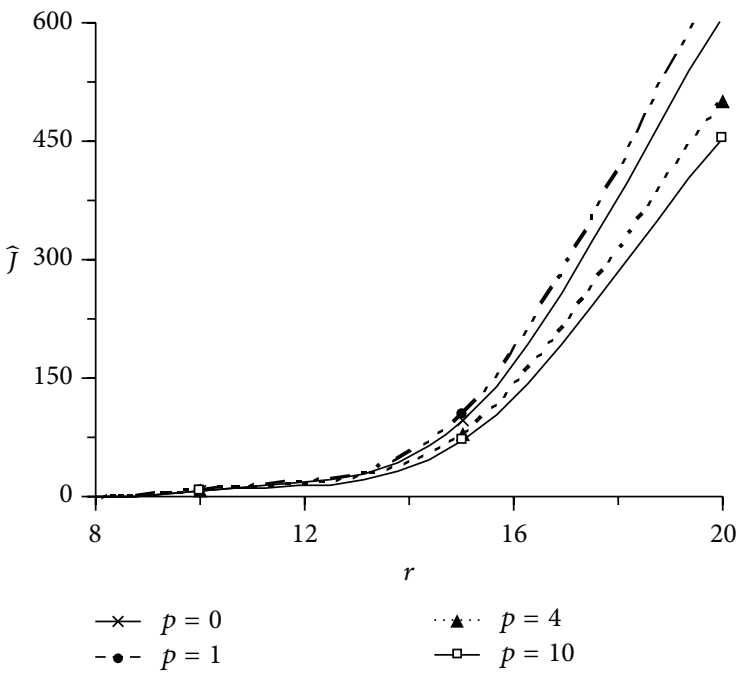

(b)

FIGURE 1: Effect of the thickness ratio $r$ and the index $p$ on curve for (a) the control force $\bar{q}_{\text {opt }}$, (b) for the energy $\widehat{J}$, with $h=0.02 \mathrm{~m}, a / b=1$.

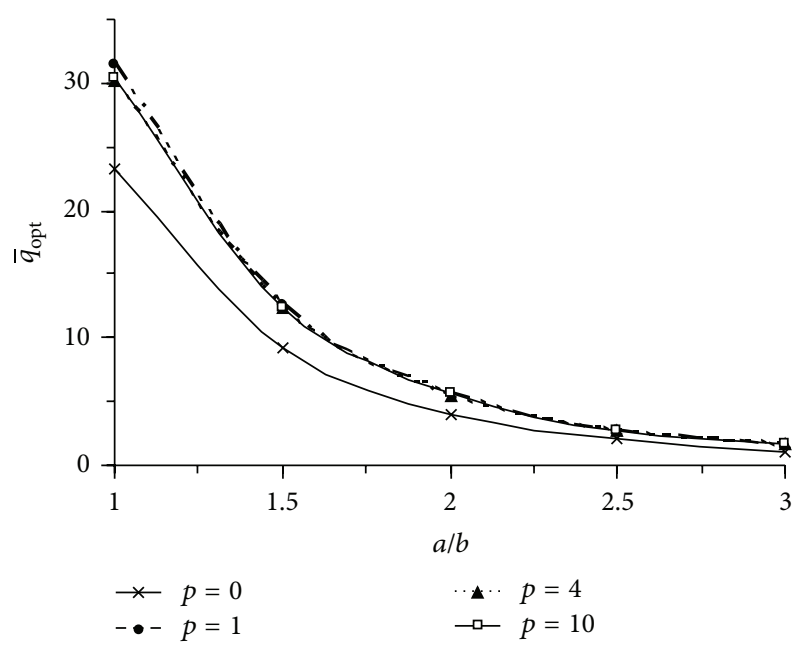

(a)

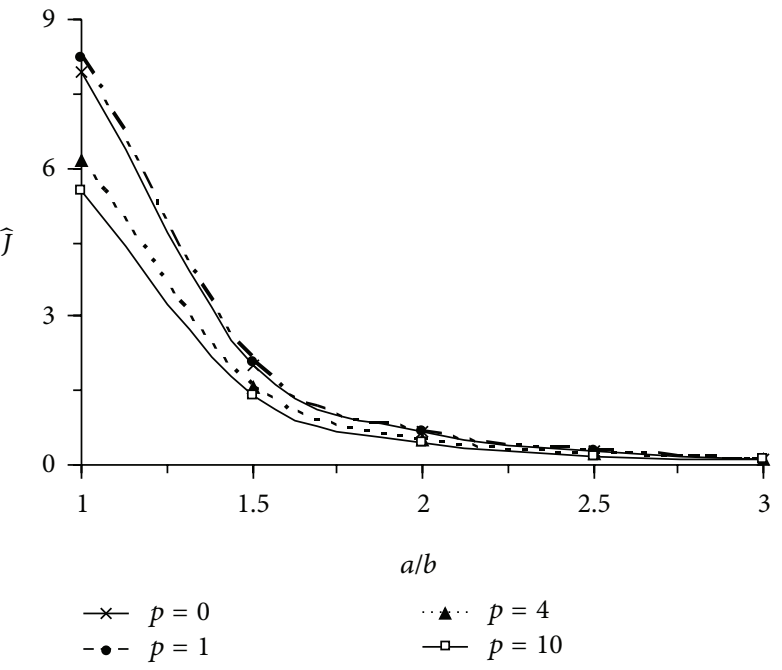

(b)

FIGURE 2: Effect of the aspect ratio $a / b$ and the index $p$ on curves for (a) the control force $\bar{q}_{\text {opt }}$, (b) for the energy $\widehat{J}$, with $h=0.02 \mathrm{~m}, r=10$.

the errors reach more than $22 \%$ for plates with $a / b=1$ and $p=10$. This means that the effect of the normal strain is weak for thin FG plates with $r<10$. Moreover, the inclusion of the effect of normal strain in the formulation with the cases $p>1$ is required for obtaining more accurate results. This may be explained by the fact that, with increasing the index $p$, the homogeneity of the plate differs rapidly through the plate thickness. This requires the inclusion of the normal strain effect in the formulation to give more accurate description for the deformation process through the thickness. Table 3 contains similar numerical results as in Tables 1 and 2 for second mode $m=2, n=1$. These results show that the relative errors increase with the increase of the mode numbers $m$ and $n$ confirming the importance of the inclusion of the normal strain effect in the formulation of the problem to obtain more accurate results. In general, the effect of the normal strain on the accuracy of the numerical results of the total energy $J$ is less significant than that on the control force $q$.

Figures 1 and 2 contain curves for the controlled force $q$ and the total energy $\widehat{J}(=100 J)$ versus the ratios $r$ and $a / b$ for different values of index $p$ for SSSS plates. These curves show that the volume fraction profile through the plate thickness has a significant effect on the control force and controlled energy, particularly for FG plates with $r \geq 15$ and square plates. Moreover, the curves show the high sensitivity of the total energy and control force of the FG plates versus the 


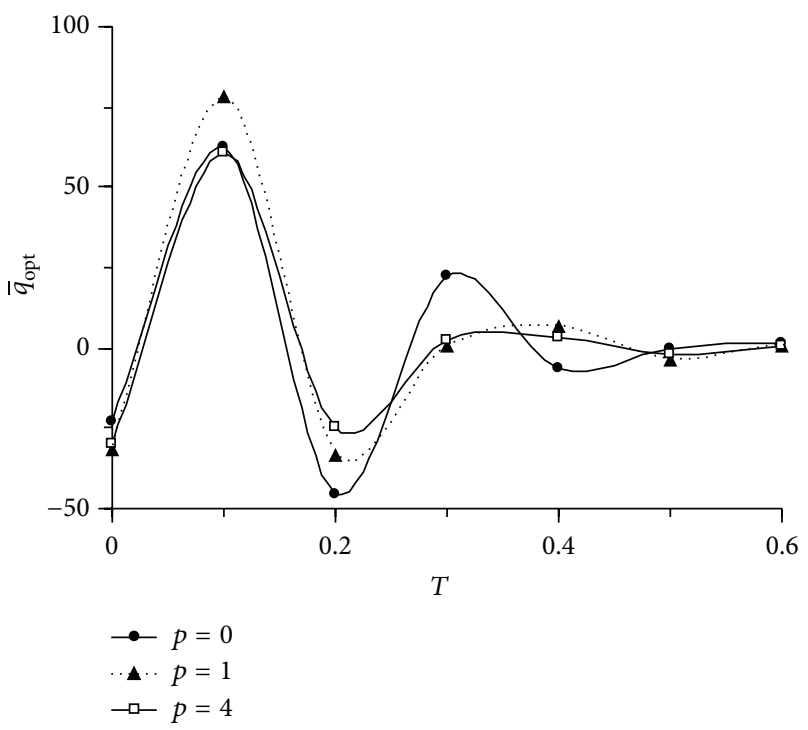

(a)

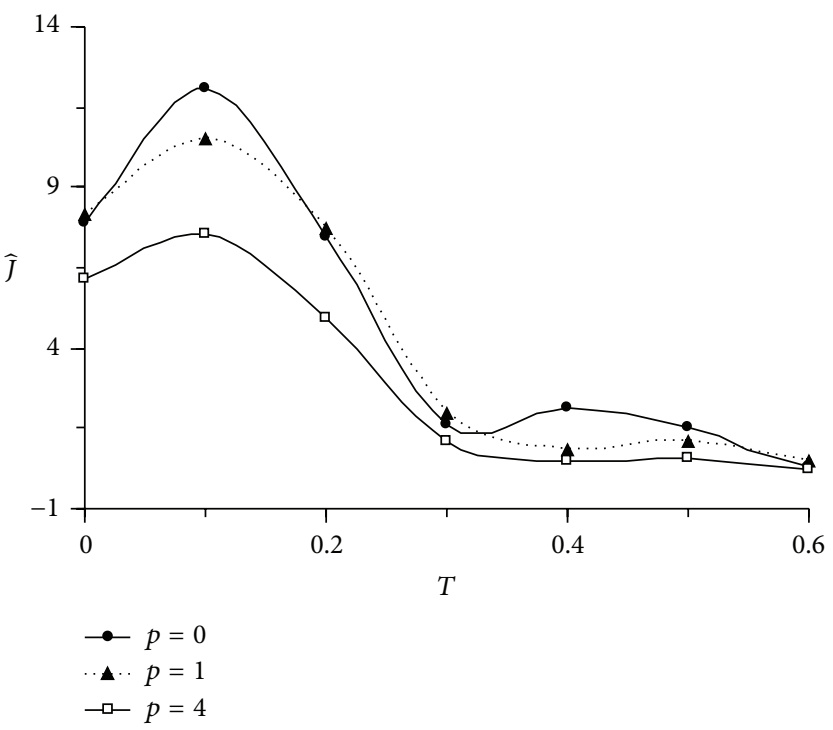

(b)

FIGURE 3: Effect of the index $p$ on curves for (a) the optimal control force $\bar{q}_{\text {opt }}$, (b) for the energy $\widehat{J}$, against the time $T, h=0.02 \mathrm{~m}$ and $a / b=1$.

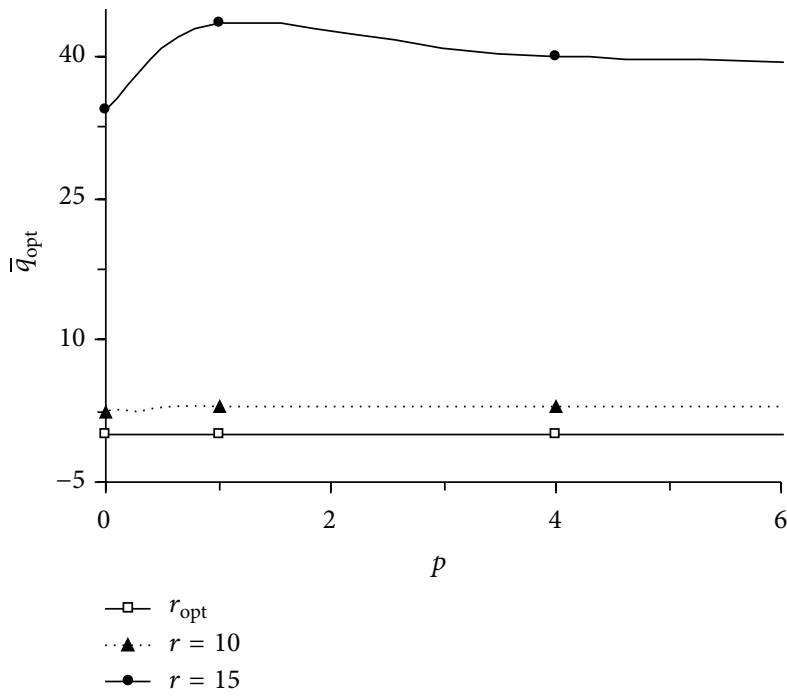

(a)

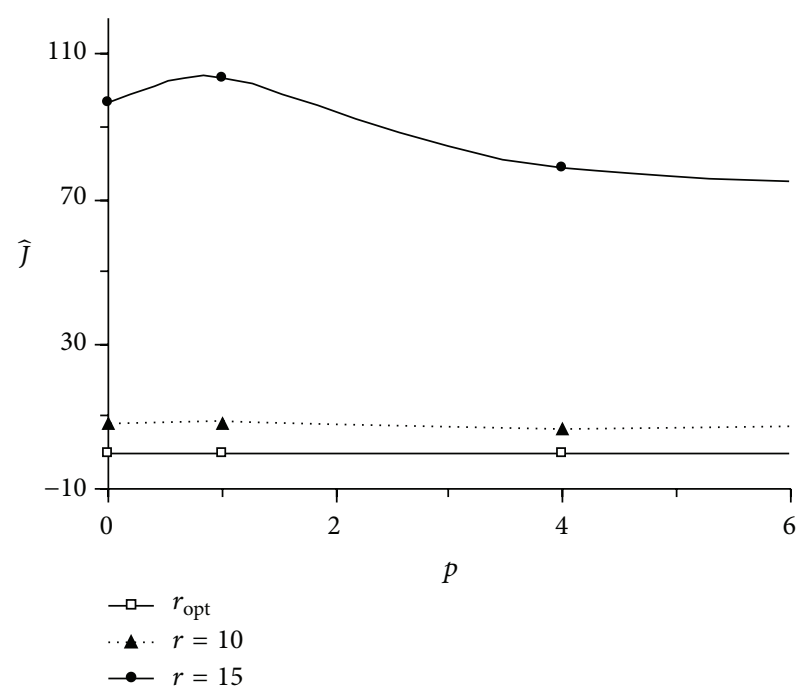

(b)

FIGURE 4: Effect of the thickness ratio $r$ and the index $p$ on curves of the control force $\bar{q}_{\text {opt }}$ and the energy $\widehat{J}$ for SSSS plates with $a / b=1$.

change of ratios $r, a / b$ and the index $p$. This indicates that these design variables may play an important role in reducing the vibrational response of the FG plates. Figure 3 shows $q$-curves and $\widehat{J}$-curves against the time parameter $T$. These curves show that the present design control procedure reduces the expenditure of the control energy and the time of the controlling process. Figure 4 contains curves for the control force $q$ and the total energy $\widehat{J}$ against the index number $p$ for different values of the thickness ratio $r$. These curves confirm the previous discussion on the efficiency of the present procedure in damping process, where the use of the thickness ratio $r$ as design variable reduces significantly the level of the total energy and decreases the consumed control energy. Figure 5 contains $q$-curves and $\widehat{J}$-curves against the volume fraction $V_{1}^{+}$. Note that the design procedure over the plate thickness is more efficient than that over the volume fractional profile, while the design procedure over both $r$ and $p$ is the most efficient. In general the present control and design procedure strongly improves the performance of the FG plates. 


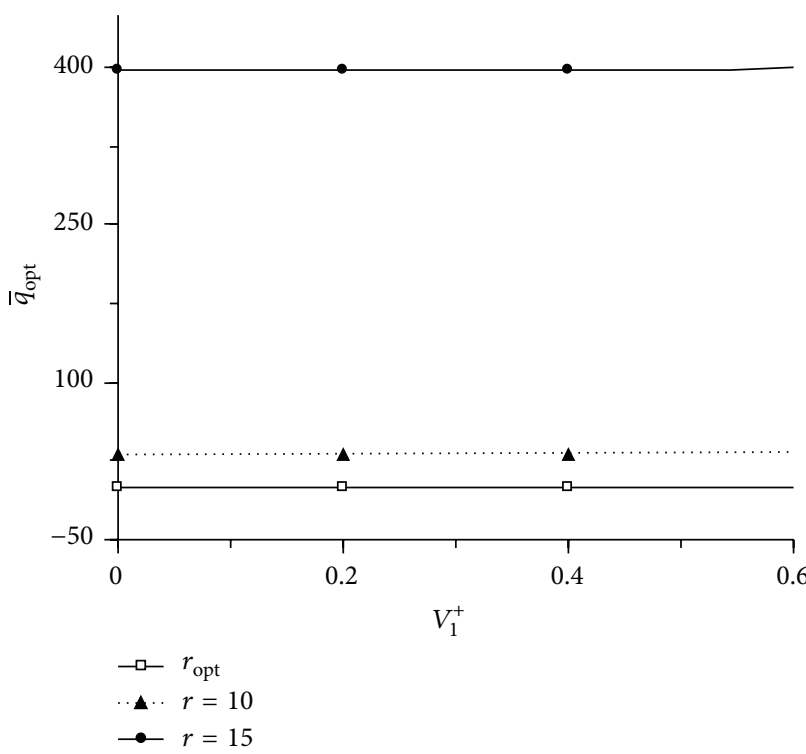

(a)

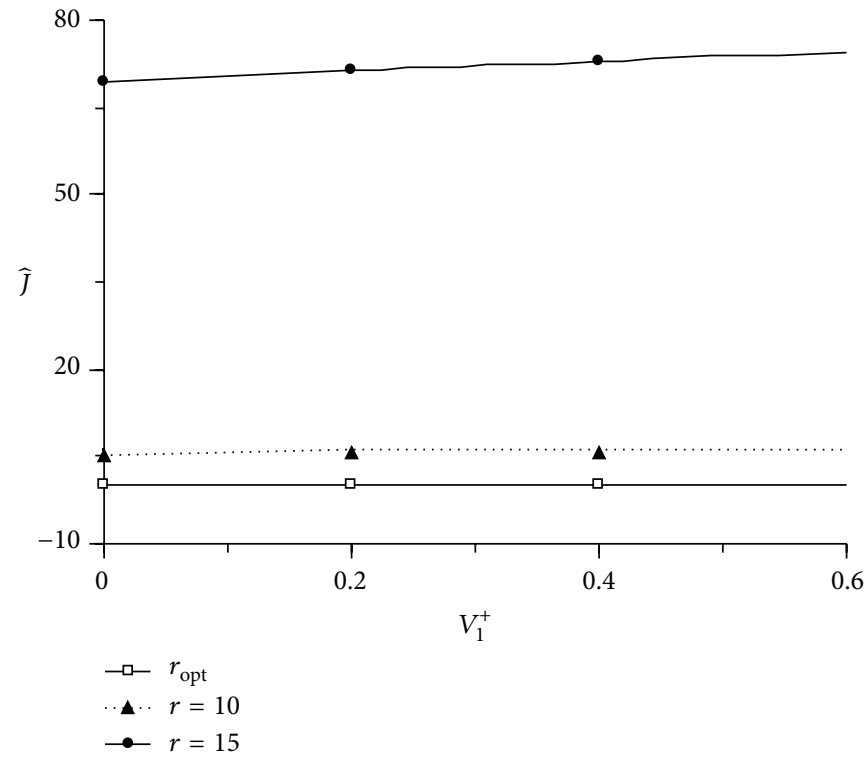

(b)

FIGURE 5: Effect of the thickness ratio $r$ and volume fraction $V_{1}^{+}$on curves of the control force $\bar{q}_{\text {opt }}$ and the energy $\widehat{J}$ for SSSS plates with $p=4$ and $a / b=1$.

\section{Conclusion}

A structural and control optimization procedure is presented to minimize the vibrational response of FG plates. A firstorder plate theory including the normal strain and shear deformation effects is used. Optimal level of closed-loop control force, plate thickness, and the volume fractional index are determined. The influence of the normal strain effect on the accuracy of the obtained results is illustrated. It is found that the inclusion of the normal stain effect is required, so that neglecting the normal stain effect leads to errors reaching more than $22 \%$ for square plates with homogeneity index $p>1$. The presented results show that the present control and design procedure has a high efficiency in improving the performance of the FG plates.

\section{Appendices}

\section{A. The Definition of the Parameters in $(6 a)$ and $(6 b)$}

The stiffness coefficients $A_{i j}, B_{i j}$, and $D_{i j}$ in (6a) and (6b) are

$$
\begin{gathered}
\left(A_{i j}, B_{i j}, D_{i j}\right)=\int_{-h / 2}^{h / 2} a_{i j}\left(\frac{1}{h^{2}}, \frac{12 x_{3}}{h^{4}}, \frac{144 x_{3}^{2}}{h^{6}}\right) d x_{3} \\
(i, j=1,2,6), \\
\left(A_{i i}\right)=\int_{-h / 2}^{h / 2} \frac{9}{4 h^{2}} a_{i i}\left(1-\left(\frac{x_{3}}{h / 2}\right)^{2}\right)^{2} d x_{3}, \quad(i=4,5),
\end{gathered}
$$

$$
\begin{gathered}
\left(A_{i 3}, B_{i 3}, \bar{B}_{i 3}, D_{i 3}\right) \\
=\int_{-h / 2}^{h / 2} a_{i 3}\left(\frac{3}{2 h^{2}}, \frac{30 x_{3}}{h^{4}}, \frac{18 x_{3}}{h^{4}}, \frac{360 x_{3}^{2}}{h^{6}}\right) \\
\times\left(1-\left(\frac{x_{3}}{h / 2}\right)^{2}\right) d x_{3}, \quad(i, j=1,2), \\
\left(A_{33}, B_{33}, D_{33}\right)=\int_{-h / 2}^{h / 2} a_{33}\left(\frac{9}{4 h^{2}}, \frac{45 x_{3}}{h^{4}}, \frac{900 x_{3}^{2}}{h^{6}}\right) \\
\times\left(1-\left(\frac{x_{3}}{h / 2}\right)^{2}\right)^{2} d x_{3},
\end{gathered}
$$$$
\left(F_{i 3}, K_{i 3}\right)
$$$$
=\int_{-h / 2}^{h / 2} a_{i 3}\left(\frac{1}{h}, \frac{12 x_{3}}{h^{3}}\right)
$$$$
\times\left\{\frac{q_{1}}{4}\left[\left(\frac{x_{3}}{h / 2}\right)^{2}-\left(\frac{x_{3}}{h / 2}\right)-2\right]\right.
$$$$
\times\left[1+\left(\frac{x_{3}}{h / 2}\right)\right]
$$$$
+\frac{q_{2}}{4}\left[\left(\frac{x_{3}}{h / 2}\right)^{2}+\left(\frac{x_{3}}{h / 2}\right)-2\right]
$$$$
\left.\times\left[1-\left(\frac{x_{3}}{h / 2}\right)\right]\right\} d x_{3}, \quad(i=1,2),
$$ 


$$
\begin{gathered}
\left(F_{33}, K_{33}\right) \\
=\int_{-h / 2}^{h / 2} a_{33}\left(\frac{3}{2 h}, \frac{30 x_{3}}{h^{3}}\right) \\
\times\left(\frac{q_{1}}{4}\left[\left(\frac{x_{3}}{h / 2}\right)^{2}-\left(\frac{x_{3}}{h / 2}\right)-2\right]\left(1+\frac{x_{3}}{h / 2}\right)\right. \\
+\frac{q_{2}}{4}\left[\left(\frac{x_{3}}{h / 2}\right)^{2}+\left(\frac{x_{3}}{h / 2}\right)-2\right] \\
\left.\quad \times\left(1-\frac{x_{3}}{h / 2}\right)\right) d x_{3}, \\
H_{1}=\int_{-h / 2}^{h / 2} \frac{3 a_{55}}{2 h}\left[1-\left(\frac{x_{3}}{h / 2}\right)^{2}\right] \\
\times\left\{\frac{P_{1}^{+}}{4}\left[1-3\left(\frac{x_{3}}{h / 2}\right)\right]\left[1+\left(\frac{x_{3}}{h / 2}\right)\right]\right. \\
\left.-\frac{P_{j 3}^{-}}{4\left(x_{1}, x_{2}, t\right)}, \quad S_{j+3}=\frac{P_{2}^{-}}{4}\left[1+3\left(\frac{x_{3}}{h / 2}\right)\right]\left[1-\left(\frac{x_{3}}{h / 2}\right)\right]\right\} d x_{3}, \\
\times\left\{\frac{P_{2}^{+}}{4}\left[1-3\left(\frac{x_{3}}{h / 2}\right)\right]\left[1+\left(\frac{x_{3}}{h / 2}\right)\right]\left[1-\left(\frac{x_{3}}{h / 2}\right)\right]\right\} d x_{3}, \\
H_{2}=\int_{-h / 2}^{h / 2} \frac{3 a_{44}}{2 h}\left[1-\left(\frac{x_{3}}{h / 2}\right)^{2}\right]
\end{gathered}
$$

\section{B. The Definition of the Parameters}

in (12) and (14)

The coefficients $U_{1 i}, U_{2 i}, \Psi_{1 i}, \Psi_{2 i}, W_{0 i}, W_{1 i}, W_{2 i}, Q_{i}$ in (12) are

$$
\begin{aligned}
& U_{11}=A_{11}^{*} e_{3}+A_{66}^{*} e_{1}, \\
& U_{21}=\left(A_{12}^{*}+A_{66}^{*}\right) e_{1}, \\
& \Psi_{11}=B_{11}^{*} e_{3}+B_{66}^{*} e_{1}, \\
& \Psi_{21}=\left(B_{12}^{*}+B_{66}^{*}\right) e_{1}, \\
& U_{12}=\left(A_{12}^{*}+A_{66}^{*}\right) e_{4}, \\
& U_{22}=A_{66}^{*} e_{4}+A_{22}^{*} e_{6},
\end{aligned}
$$

$$
\begin{aligned}
& \Psi_{12}=\left(B_{12}^{*}+B_{66}^{*}\right) e_{4}, \\
& \Psi_{22}=B_{22}^{*} e_{6}+B_{66}^{*} e_{4}, \\
& U_{14}=B_{11}^{*} e_{3}+B_{66}^{*} e_{1}, \\
& U_{24}=\left(B_{12}^{*}+B_{66}^{*}\right) e_{1}, \\
& \Psi_{14}=f_{11}^{*} e_{3}+f_{66}^{*} e_{1}-A_{55}^{*} e_{2}, \\
& \Psi_{24}=\left(f_{12}^{*}+f_{66}^{*}\right) e_{1}, \\
& U_{15}=\left(B_{12}^{*}+B_{66}^{*}\right) e_{4}, \\
& U_{25}=B_{66}^{*} e_{4}+B_{22}^{*} e_{6}, \\
& \Psi_{15}=\left(f_{12}^{*}+f_{66}^{*}\right) e_{4}, \\
& \Psi_{25}=f_{66}^{*} e_{4}+f_{22}^{*} e_{6}-A_{44}^{*} e_{5}, \\
& U_{16}=-A_{13}^{*} e_{7}, \quad U_{26}=-A_{23}^{*} e_{8}, \\
& \Psi_{16}=-b_{13}^{*} e_{7}, \quad \Psi_{26}=-b_{23}^{*} e_{8}, \\
& U_{17}=-2 B_{13}^{*} e_{7}, \quad U_{27}=-2 B_{23}^{*} e_{8}, \\
& \Psi_{17}=\left(\frac{h^{2} A_{55}^{*}}{20}-2 f_{13}^{*}\right) e_{7}, \\
& \Psi_{27}=\left(\frac{h^{2} A_{44}^{*}}{20}-2 f_{23}^{*}\right) e_{8}, \\
& W_{04}=-A_{55}^{*} e_{2}, \quad W_{11}=\alpha A_{13}^{*} e_{2}, \\
& W_{21}=2 \beta B_{13}^{*} e_{2}, \quad \Psi_{13}=A_{55}^{*} e_{7}, \\
& \Psi_{23}=A_{44}^{*} e_{8}, \quad W_{05}=-A_{44}^{*} e_{5}, \\
& W_{12}=\alpha A_{23}^{*} e_{5}, \quad W_{22}=2 \beta B_{23}^{*} e_{5}, \\
& W_{23}=\frac{\beta h^{2}\left(A_{55}^{*} e_{7}+A_{44}^{*} e_{8}\right)}{20}, \\
& W_{07}=\frac{h^{2}\left(A_{55}^{*} e_{7}+A_{44}^{*} e_{8}\right)}{20}, \\
& W_{14}=\alpha b_{13}^{*} e_{2}, \\
& W_{24}=\beta\left(2 f_{13}^{*}-\frac{A_{55}^{*} h^{2}}{20}\right) e_{2}, \\
& \bar{W}_{3}=I_{0} e_{9}, \quad W_{15}=\alpha b_{23}^{*} e_{5},
\end{aligned}
$$




$$
\begin{aligned}
& W_{25}=\beta\left(2 f_{23}^{*}-\frac{A_{44}^{*} h^{2}}{20}\right) e_{5}, \\
& \bar{W}_{6}=I_{1} e_{9}, \quad W_{16}=-\alpha A_{33}^{*} e_{9}, \\
& W_{26}=-2 \beta B_{33}^{*} e_{9}, \quad \bar{W}_{7}=I_{2} e_{9}, \\
& W_{17}=-2 \alpha B_{33}^{*} e_{9}, \\
& W_{27}=\beta\left[\frac{h^{4}\left(A_{55}^{*} e_{7}+A_{44}^{*} e_{8}\right)}{400}-4 f_{33}^{*} e_{9}\right], \\
& U_{13}=U_{23}=W_{01}=W_{02}=W_{13}=W_{06}=0, \\
& Q_{1}=\left(A_{11}^{*} S_{1}+A_{12}^{*} S_{2}+A_{13}^{*} S_{3}+B_{11}^{*} S_{4}+B_{12}^{*} S_{5}+B_{13}^{*} S_{6}\right) e_{2} \text {, } \\
& Q_{2}=\left(A_{12}^{*} S_{1}+A_{22}^{*} S_{2}+A_{23}^{*} S_{3}+B_{12}^{*} S_{4}+B_{22}^{*} S_{5}+B_{23}^{*} S_{6}\right) e_{5} \text {, } \\
& Q_{4}=\left(B_{11}^{*} S_{1}+B_{12}^{*} S_{2}+B_{13}^{*} S_{3}+f_{11}^{*} S_{4}+f_{12}^{*} S_{5}+f_{13}^{*} S_{6}\right) e_{2}, \\
& Q_{5}=\left(B_{12}^{*} S_{1}+B_{22}^{*} S_{2}+B_{23}^{*} S_{3}+f_{12}^{*} S_{4}+f_{22}^{*} S_{5}+f_{23}^{*} S_{6}\right) e_{5}, \\
& Q_{6}=-\left(A_{13}^{*} S_{1}+A_{23}^{*} S_{2}+A_{33}^{*} S_{3}+b_{13}^{*} S_{4}\right. \\
& \left.+b_{23}^{*} S_{5}+B_{33}^{*} S_{6}+h\right) e_{9} \\
& Q_{7}=-2\left(B_{13}^{*} S_{1}+B_{23}^{*} S_{2}+B_{33}^{*} S_{3}+f_{13}^{*} S_{4}\right. \\
& \left.+f_{23}^{*} S_{5}+f_{33}^{*} S_{6}+\frac{9 h^{2}}{40}\right) e_{9} \\
& Q_{3}=-e_{9}, \\
& \Delta_{1}=\Delta_{41} \Psi_{13}+\Delta_{51} \Psi_{23}+\Delta_{71} W_{23}-\Delta_{0} W_{03} \text {, } \\
& \Delta_{2}=\Delta_{42} \Psi_{13}+\Delta_{52} \Psi_{23}+\Delta_{72} W_{23}+\Delta_{0} e_{9}, \\
& \Delta_{3}=\Delta_{0} \bar{W}_{3}-\Delta_{43} \Psi_{3}-\Delta_{53} \Phi_{3}-\Delta_{73} W_{23}, \\
& \Delta_{0}=\left[\begin{array}{llllll}
P_{11} & P_{22} & P_{33} & P_{44} & P_{55} & P_{66}
\end{array}\right], \\
& \Delta_{11}=\left[\begin{array}{llllll}
\Gamma & P_{22} & P_{33} & P_{44} & P_{55} & P_{66}
\end{array}\right], \\
& \Delta_{12}=\left[\begin{array}{llllll}
\zeta & P_{22} & P_{33} & P_{44} & P_{55} & P_{66}
\end{array}\right], \\
& \Delta_{21}=\left[\begin{array}{llllll}
P_{11} & \Gamma & P_{33} & P_{44} & P_{55} & P_{66}
\end{array}\right], \\
& \Delta_{22}=\left[\begin{array}{llllll}
P_{11} & \zeta & P_{33} & P_{44} & P_{55} & P_{66}
\end{array}\right], \\
& \Delta_{41}=\left[\begin{array}{llllll}
P_{11} & P_{22} & \Gamma & P_{44} & P_{55} & P_{66}
\end{array}\right], \\
& \Delta_{42}=\left[\begin{array}{llllll}
P_{11} & P_{22} & \zeta & P_{44} & P_{55} & P_{66}
\end{array}\right],
\end{aligned}
$$

$\Delta_{51}=\left[\begin{array}{llllll}P_{11} & P_{22} & P_{33} & \Gamma & P_{55} & P_{66}\end{array}\right]$,

$\Delta_{52}=\left[\begin{array}{llllll}P_{11} & P_{22} & P_{33} & \zeta & P_{55} & P_{66}\end{array}\right]$,

$\Delta_{61}=\left[\begin{array}{llllll}P_{11} & P_{22} & P_{33} & P_{44} & \Gamma & P_{66}\end{array}\right]$,

$\Delta_{62}=\left[\begin{array}{llllll}P_{11} & P_{22} & P_{33} & P_{44} & \zeta & P_{66}\end{array}\right]$,

$\Delta_{71}=\left[\begin{array}{llllll}P_{11} & P_{22} & P_{33} & P_{44} & P_{55} & \Gamma\end{array}\right]$,

$\Delta_{72}=\left[\begin{array}{llllll}P_{11} & P_{22} & P_{33} & P_{44} & P_{55} & \zeta\end{array}\right]$,

$\Delta_{13}=\left[\begin{array}{llllll}\chi & P_{22} & P_{33} & P_{44} & P_{55} & P_{66}\end{array}\right]$,

$\Delta_{23}=\left[\begin{array}{llllll}P_{11} & \chi & P_{33} & P_{44} & P_{55} & P_{66}\end{array}\right]$,

$\Delta_{43}=\left[\begin{array}{llllll}P_{11} & P_{22} & \chi & P_{44} & P_{55} & P_{66}\end{array}\right]$,

$\Delta_{63}=\left[\begin{array}{llllll}P_{11} & P_{22} & P_{33} & P_{44} & \chi & P_{66}\end{array}\right]$,

$\Delta_{53}=\left[\begin{array}{llllll}P_{11} & P_{22} & P_{33} & \chi & P_{55} & P_{66}\end{array}\right]$,

$\Delta_{73}=\left[\begin{array}{llllll}P_{11} & P_{22} & P_{33} & P_{44} & P_{55} & \chi\end{array}\right]$,

$P_{11}=\left[\begin{array}{llllll}U_{11} & U_{12} & U_{14} & U_{15} & U_{16} & U_{17}\end{array}\right]^{T}$,

$P_{22}=\left[\begin{array}{llllll}U_{21} & U_{22} & U_{24} & U_{25} & U_{26} & U_{12}\end{array}\right]^{T}$,

$P_{33}=\left[\begin{array}{lllllll}\Psi_{11} & \Psi_{12} & \Psi_{14} & \Psi_{15} & \Psi_{16} & \Psi_{17}\end{array}\right]^{T}$,

$P_{44}=\left[\begin{array}{lllllll}\Psi_{21} & \Psi_{22} & \Psi_{24} & \Psi_{25} & \Psi_{26} & \Psi_{27}\end{array}\right]^{T}$,

$P_{55}=\left[\begin{array}{llllll}W_{11} & W_{12} & W_{14} & W_{15} & W_{16} & W_{17}\end{array}\right]^{T}$,

$P_{66}=\left[\begin{array}{llllll}W_{21} & W_{22} & W_{24} & W_{25} & W_{26} & W_{27}\end{array}\right]^{T}$,

$\Gamma=\left[\begin{array}{llllll}0 & 0 & W_{04} & 0 & W_{06} & W_{07}\end{array}\right]^{T}$,

$\zeta=\left[\begin{array}{llllll}Q_{1} & Q_{2} & Q_{4} & Q_{5} & Q_{6} & Q_{7}\end{array}\right]^{T}$, $\chi=\left[\begin{array}{llllll}0 & 0 & 0 & 0 & \bar{W}_{6} & \bar{W}_{7}\end{array}\right]^{T}$.

The coefficients $K_{i}(i=1,2, \ldots, 6)$ in (14) are

$$
\begin{gathered}
K_{1}=L_{1} e_{2}+L_{2} e_{5}+L_{3} e_{7}+L_{4} e_{8}+L_{5} e_{9} \\
+L_{6} e_{10}+L_{7} e_{11}+L_{8} e_{12}+L_{9} e_{13}, \\
K_{2}=L_{10} e_{2}+L_{11} e_{5}+L_{12} e_{7}+L_{13} e_{8}+L_{14} e_{9} \\
+L_{15} e_{10}+L_{16} e_{11}+L_{17} e_{12}+L_{18} e_{13},
\end{gathered}
$$




$$
\begin{aligned}
& K_{3}=L_{19} e_{2}+L_{20} e_{5}+L_{21} e_{7}+L_{22} e_{8}+L_{23} e_{9} \\
& L_{6}=\left(800 f_{12} \Delta_{41} \Delta_{51}+800 A_{12} \Delta_{11} \Delta_{21}\right. \\
& +L_{24} e_{10}+L_{25} e_{11}+L_{26} e_{12}+L_{27} e_{13} \text {, } \\
& \left.+800 B_{12} \Delta_{11} \Delta_{51}+800 B_{12} \Delta_{41} \Delta_{21}\right)\left(800 \Delta_{0}^{2}\right)^{-1}, \\
& K_{4}=L_{28} e_{2}+L_{29} e_{5}+L_{30} e_{9}, \\
& K_{5}=L_{31} e_{2}+L_{32} e_{5}+L_{33} e_{9}, \\
& K_{6}=L_{34} e_{2}+L_{35} e_{5}+L_{36} e_{9}, \\
& \left(e_{1}, e_{2}, e_{3}\right) \\
& =\int_{0}^{b} \int_{0}^{a}\left(X_{1, x_{1}} X_{2, x_{2} x_{2}}, X_{1, x_{1}} X_{2}, X_{1, x_{1} x_{1} x_{1}} X_{2}\right) \\
& \times X_{1, x_{1}} X_{2} d x_{1} d x_{2}, \\
& \left(e_{4}, e_{5}, e_{6}\right) \\
& =\int_{0}^{b} \int_{0}^{a}\left(X_{1, x_{1} x_{1}} X_{2, x_{2}}, X_{1} X_{2, x_{2}}, X_{1} X_{2, x_{2} x_{2} x_{2}}\right) \\
& \times X_{1} X_{2, x_{2}} d x_{2} d x_{2}, \\
& L_{7}=\frac{m_{1} A_{66}+m_{2} B_{66}+m_{3} f_{66}}{800 \Delta_{0}^{2}} \\
& L_{8}=\frac{\left(400 f_{22} \Delta_{51}^{2}+800 B_{22} \Delta_{51} \Delta_{21}+400 A_{22} \Delta_{21}^{2}\right)}{800 \Delta_{0}^{2}} \\
& L_{9}=\left(40 A_{11} \Delta_{11}^{2}+800 B_{11} \Delta_{41} \Delta_{11}\right. \\
& \left.+400 f_{11} \Delta_{41}^{2}-800 A_{55} \Delta_{0} \Delta_{41}\right)\left(800 \Delta_{0}^{2}\right)^{-1}, \\
& L_{10}=\left(\left(40 h^{2} \Delta_{0} \Delta_{72}-40 h^{2} \Delta_{41} \Delta_{72}+800 \Delta_{0} \Delta_{42}\right.\right. \\
& -800 \Delta_{41} \Delta_{42}-40 h^{2} \Delta_{42} \Delta_{71} \\
& \left.\left.-2 h^{4} \Delta_{71} \Delta_{72}\right) A_{55}\right)\left(800 \Delta_{0}^{2}\right)^{-1} \\
& \left(e_{7}, e_{8}, e_{9}, e_{10}\right) \\
& =\int_{0}^{b} \int_{0}^{a}\left(X_{2, x_{2} x_{2}} X_{2}, X_{1} X_{2, x_{2} x_{2}}, X_{1} X_{2}, X_{1, x_{1} x_{1}} X_{2, x_{2} x_{2}}\right) \\
& \times X_{1} X_{2} d x_{1} d x_{2} \\
& \left(e_{11}, e_{12}, e_{13}\right) \\
& =\int_{0}^{b} \int_{0}^{a}\left(X_{1, x_{1}}^{2} X_{2, x_{2}}^{2}, X_{1}^{2} X_{2, x_{2} x_{2}}^{2}, X_{1, x_{1} x_{1}}^{2} X_{2}^{2}\right) d x_{1} d x_{2} \\
& L_{1}=\left(\left(h^{4} \Delta_{71}^{2}+400 \Delta_{41}^{2}+400 \Delta_{0}^{2}+40 \Delta_{41}^{2}\right.\right. \\
& \left.\left.+40 h^{2} \Delta_{41} \Delta_{71}-40 h^{2} \Delta_{0} \Delta_{71}\right) A_{55}\right)\left(800 \Delta_{0}^{2}\right)^{-1}, \\
& L_{2}=\left(\left(h^{4} \Delta_{71}^{2}+400 \Delta_{51}^{2}+400 \Delta_{0}^{2}+40 \Delta_{51}^{2}+40 h^{2} \Delta_{51} \Delta_{71}\right.\right. \\
& \left.\left.-40 h^{2} \Delta_{0} \Delta_{71}-800 h^{2} \Delta_{0} \Delta_{51}\right) A_{44}\right)\left(800 \Delta_{0}^{2}\right)^{-1}, \\
& L_{3}=\left(1600 f_{13} \Delta_{41} \Delta_{71}+1600 B_{13} \Delta_{11} \Delta_{71}\right. \\
& \left.+800 b_{13} \Delta_{41} \Delta_{61}+800 A_{13} \Delta_{11} \Delta_{61}\right)\left(800 \Delta_{0}^{2}\right)^{-1}, \\
& L_{4}=\left(1600 f_{23} \Delta_{51} \Delta_{71}+1600 B_{23} \Delta_{21} \Delta_{71}\right. \\
& \left.+800 b_{23} \Delta_{51} \Delta_{61}+800 A_{23} \Delta_{21} \Delta_{61}\right)\left(800 \Delta_{0}^{2}\right)^{-1}, \\
& L_{5}=\frac{\left(1600 f_{33} \Delta_{71}^{2}+1600 B_{33} \Delta_{61} \Delta_{71}+400 A_{33} \Delta_{61}^{2}\right)}{800 \Delta_{0}^{2}}, \\
& L_{11}=\left(\left(40 h^{2} \Delta_{0} \Delta_{72}-40 h^{2} \Delta_{52} \Delta_{71}+800 \Delta_{0} \Delta_{52}\right.\right. \\
& -800 \Delta_{51} \Delta_{52}-40 h^{2} \Delta_{51} \Delta_{72} \\
& \left.\left.-2 h^{4} \Delta_{71} \Delta_{72}\right) A_{44}\right)\left(800 \Delta_{0}^{2}\right)^{-1} \\
& L_{12}=\frac{m_{4} \Delta_{0} \Delta_{11}+m_{5} \Delta_{0} \Delta_{41}+m_{6}}{800 \Delta_{0}^{2}}, \\
& L_{13}=\frac{m_{7} \Delta_{0} \Delta_{21}+m_{8} \Delta_{0} \Delta_{51}+m_{9}}{800 \Delta_{0}^{2}}, \\
& L_{14}=\frac{m_{10} \Delta_{0} \Delta_{61}+m_{11} \Delta_{0} \Delta_{71}+m_{12}}{800 \Delta_{0}^{2}}, \\
& L_{15}=\frac{m_{13} A_{12}+m_{14} B_{12}+m_{15} f_{12}}{800 \Delta_{0}^{2}}, \\
& L_{16}=\frac{m_{16} A_{66}+m_{17} B_{66}+m_{18} f_{66}}{800 \Delta_{0}^{2}}, \\
& L_{17}=\left(-800\left(\Delta_{21} \Delta_{22} A_{22}+\left(\Delta_{51} \Delta_{22}+\Delta_{52} \Delta_{21}\right) B_{22}\right.\right. \\
& \left.\left.+\Delta_{51} \Delta_{52} f_{22}\right)\right)\left(800 \Delta_{0}^{2}\right)^{-1} \\
& L_{18}=\left(-800\left(\Delta_{11} \Delta_{12} A_{11}+\left(\Delta_{41} \Delta_{12}+\Delta_{42} \Delta_{11}\right) B_{11}\right.\right. \\
& \left.\left.+\Delta_{41} \Delta_{42} f_{11}\right)\right)\left(800 \Delta_{0}^{2}\right)^{-1} \\
& L_{19}=\frac{\left(400 \Delta_{42}^{2}+40 h^{2} \Delta_{42} \Delta_{72}+h^{4} \Delta_{72}^{2}\right) A_{55}}{800 \Delta_{0}^{2}},
\end{aligned}
$$




$$
\begin{aligned}
& L_{20}=\frac{\left(400 \Delta_{52}^{2}+40 h^{2} \Delta_{52} \Delta_{72}+h^{4} \Delta_{72}^{2}\right) A_{44}}{800 \Delta_{0}^{2}}, \\
& L_{21}=\frac{m_{19} \Delta_{0} \Delta_{12}+m_{20} \Delta_{0} \Delta_{42}+m_{21}}{800 \Delta_{0}^{2}} \\
& L_{22}=\frac{m_{22} \Delta_{0} \Delta_{22}+m_{23} \Delta_{0} \Delta_{52}+m_{24}}{800 \Delta_{0}^{2}} \\
& L_{23}=\frac{-\left(m_{10} \Delta_{0} \Delta_{62}+m_{11} \Delta_{0} \Delta_{72}+m_{25}\right)}{800 \Delta_{0}^{2}}, \\
& L_{24}=\left(8 0 0 \left(\Delta_{12} \Delta_{22} A_{12}+\left(\Delta_{12} \Delta_{22}+\Delta_{42} \Delta_{22}\right) B_{11}\right.\right. \\
& \left.\left.+\Delta_{42} \Delta_{52} f_{12}\right)\right)\left(800 \Delta_{0}^{2}\right)^{-1} \text {, } \\
& L_{25}=\frac{m_{26} A_{66}+m_{27} B_{66}+m_{28} f_{66}}{800 \Delta_{0}^{2}}, \\
& L_{26}=\frac{400\left(\Delta_{22}^{2} A_{22}+2 \Delta_{22} \Delta_{52} B_{22}+f_{22} \Delta_{52}^{2}\right)}{800 \Delta_{0}^{2}}, \\
& L_{27}=\frac{400\left(\Delta_{12}^{2} A_{11}+2 \Delta_{12} \Delta_{42} B_{22}+f_{11} \Delta_{42}^{2}\right)}{800 \Delta_{0}^{2}}, \\
& m_{1}=400 \Delta_{11}^{2}+800 \Delta_{11} \Delta_{21}+400 \Delta_{21}^{2} \\
& m_{2}=800 \Delta_{51} \Delta_{21}+800 \Delta_{51} \Delta_{11} \\
& +800 \Delta_{41} \Delta_{21}+800 \Delta_{41} \Delta_{11} \\
& m_{3}=400 \Delta_{51}^{2}+400 \Delta_{41}^{2}+800 \Delta_{41} \Delta_{51} \\
& m_{4}=400\left(A_{11} S 1+A_{12} S 2+A_{13} S 3\right. \\
& \left.+B_{11} S 4+B_{12} S 5+B_{13} S 6\right) \\
& m_{5}=400\left(B_{11} S 1+B_{12} S 2+b_{13} S 3\right. \\
& \left.+f_{11} S 4+f_{12} S 5+f_{13} S 6\right), \\
& m_{6}=-800\left[\left(\Delta_{62} \Delta_{11}+\Delta_{61} \Delta_{12}\right) A_{13}\right. \\
& +\left(\Delta_{62} \Delta_{41}+\Delta_{61} \Delta_{42}\right) b_{13} \\
& +2\left(\Delta_{72} \Delta_{11}+\Delta_{71} \Delta_{12}\right) B_{13} \\
& \left.+2\left(\Delta_{42} \Delta_{71}+\Delta_{41} \Delta_{72}\right) f_{13}\right], \\
& m_{7}=400\left(A_{12} S 1+A_{22} S 2+A_{23} S 3\right. \\
& \left.+B_{12} S 4+B_{22} S 5+B_{23} S 6\right), \\
& m_{8}=400\left(B_{12} S 1+B_{22} S 2+b_{23} S 3\right. \\
& \left.+f_{12} S 4+f_{22} S 5+f_{23} S 6\right), \\
& m_{9}=-800\left[\left(\Delta_{62} \Delta_{21}+\Delta_{61} \Delta_{22}\right) A_{23}\right. \\
& +\left(\Delta_{62} \Delta_{51}+\Delta_{61} \Delta_{52}\right) b_{23} \\
& +2\left(\Delta_{72} \Delta_{21}+\Delta_{71} \Delta_{22}\right) B_{23} \\
& \left.+2\left(\Delta_{52} \Delta_{71}+\Delta_{51} \Delta_{72}\right) f_{23}\right], \\
& m_{10}=400\left(A_{13} S 1+A_{23} S 2+A_{33} S 3\right. \\
& \left.+b_{13} S 4+b_{23} S 5+B_{33} S 6+\frac{h}{2}\right) \\
& m_{11}=800\left(B_{13} S 1+B_{23} S 2+B_{33} S 3\right. \\
& \left.+f_{13} S 4+f_{23} S 5+f_{33} S 6+\frac{h^{2}}{10}\right) \\
& m_{12}=-800\left[\Delta_{61} \Delta_{62} A_{33}+2\left(\Delta_{61} \Delta_{72}+\Delta_{71} \Delta_{62}\right) B_{33}\right. \\
& \left.+4 \Delta_{71} \Delta_{72} f_{23}\right] \\
& m_{13}=-800\left(\Delta_{11} \Delta_{22}+\Delta_{12} \Delta_{21}\right) \text {, } \\
& m_{14}=-800\left(\Delta_{41} \Delta_{22}+\Delta_{42} \Delta_{21}+\Delta_{52} \Delta_{11}+\Delta_{51} \Delta_{12}\right), \\
& m_{15}=-800\left(\Delta_{41} \Delta_{52}+\Delta_{42} \Delta_{51}\right) \text {, } \\
& m_{16}=-800\left(\Delta_{11} \Delta_{12}+\Delta_{11} \Delta_{22}+\Delta_{21} \Delta_{12}+\Delta_{21} \Delta_{22}\right), \\
& m_{17}=-800\left(\Delta_{41} \Delta_{12}+\Delta_{41} \Delta_{22}+\Delta_{42} \Delta_{11}+\Delta_{42} \Delta_{21}\right. \\
& \left.+\Delta_{51} \Delta_{12}+\Delta_{51} \Delta_{22}+\Delta_{52} \Delta_{11}+\Delta_{52} \Delta_{21}\right) \text {, } \\
& m_{18}=-800\left(\Delta_{41} \Delta_{42}+\Delta_{41} \Delta_{52}+\Delta_{42} \Delta_{51}+\Delta_{51} \Delta_{52}\right), \\
& m_{19}=-400\left(A_{11} S 1+A_{12} S 2+A_{13} S 3\right. \\
& \left.+B_{11} S 4+B_{12} S 5+B_{13} S 6\right), \\
& m_{20}=-400\left(B_{11} S 1+B_{12} S 2+b_{13} S 3\right. \\
& \left.+f_{11} S 4+f_{12} S 5+f_{13} S 6\right), \\
& m_{21}=+800\left[\Delta_{12} \Delta_{62} A_{13}+\Delta_{62} \Delta_{42} b_{13}\right. \\
& \left.+2 \Delta_{12} \Delta_{72} B_{13}+2 \Delta_{42} \Delta_{72} f_{13}\right],
\end{aligned}
$$




$$
\begin{gathered}
m_{22}=-400\left(A_{12} S 1+A_{22} S 2+A_{23} S 3\right. \\
\left.+B_{12} S 4+B_{22} S 5+B_{23} S 6\right), \\
m_{23}=-400\left(B_{12} S 1+B_{22} S 2+b_{23} S 3\right. \\
\left.+f_{12} S 4+f_{22} S 5+f_{213} S 6\right), \\
m_{24}=800\left[\Delta_{22} \Delta_{62} A_{23}+\Delta_{62} \Delta_{52} b_{23}\right. \\
\left.+2 \Delta_{22} \Delta_{72} B_{13}+2 \Delta_{52} \Delta_{72} f_{23}\right], \\
m_{25}=-800\left[\Delta_{62}^{2} A_{33}+2 \Delta_{62} \Delta_{72} B_{33}\right. \\
\left.+4 \Delta_{72}^{2} f_{33}+\mu \Delta_{0}^{2}\right], \\
m_{26}=400 \Delta_{12}^{2}+800 \Delta_{12} \Delta_{22}+400 \Delta_{22}^{2}, \\
m_{27}=800 \Delta_{52} \Delta_{12}+800 \Delta_{52} \Delta_{22} \\
+800 \Delta_{42} \Delta_{12}+800 \Delta_{42} \Delta_{12}, \\
400 \Delta_{42}^{2}+800 \Delta_{42} \Delta_{52}+400 \Delta_{52}^{2} .
\end{gathered}
$$

\section{The Definition of the Parameters in (23)}

The coefficients $a_{i}(i=1,2, \ldots, 18)$ of $(23)$ are

$$
\begin{aligned}
& a_{1}=4 K_{6} l^{6}, \\
& a_{2}=4 K_{6} l^{4}\left(l K_{2}+2 \omega^{2} K_{3}\right), \\
& a_{3}=K_{6} l^{2}\left(l K_{2}+2 \omega^{2} K_{3}\right)^{2}, \\
& a_{4}=-4 K_{3}^{3} l^{2}, \\
& a_{5}=-4 K_{3}^{3}\left(l K_{2}+2 \omega^{2} K_{3}\right), \\
& a_{6}=K_{3}^{3}\left(4 K_{1} K_{3}-K_{2}^{2}\right) \\
& a_{7}=4 K_{6} l^{6}, \quad a_{8}=-8 K_{3} K_{6} l^{4}, \\
& a_{9}=-K_{3} l^{2}\left(K_{3}^{2}+K_{2} K_{6} l-K_{3} K_{5} l\right), \\
& a_{10}=4 K_{3}^{2} K_{6} l^{2}, \\
& a_{11}=4 K_{3}^{2}\left(2 K_{3}^{2}+K_{2} K_{6} l-K_{3} K_{5} l\right), \\
& a_{3}^{2}\left(4 K_{4} K_{3}^{2}+K_{2}^{2} K_{6}-2 K_{2} K_{3} K_{5}\right),
\end{aligned}
$$

$$
\begin{aligned}
& a_{13}=4 K_{3}^{4}, \\
& a_{14}=2 K_{6} l^{4}\left(l K_{2}+2 \omega^{2} K_{3}\right), \\
& a_{15}=a_{1}, \quad a_{16}=\frac{1}{2} a_{8}, \\
& a_{17}=-2 K_{3} l^{2}\left(2 K_{3}^{2}+2 K_{2} K_{6} l-K_{3} K_{5} l+2 K_{3} K_{6} \omega^{2}\right), \\
& a_{18}=-K_{3}\left(l K_{2}+2 \omega^{2} K_{3}\right)\left(2 K_{3}^{2}+K_{2} K_{6} l-K_{3} K_{5} l\right) .
\end{aligned}
$$

\section{Conflict of Interests}

The authors declare that there is no conflict of interests regarding the publication of this paper.

\section{References}

[1] M. Yamanouchi, M. Koizumi, T. Hirai, and I. Shiota, "Functionally gradient materials forum," in Proceeding of the 1st International Symposium on Functionally Gradient Materials (FGM '90), pp. 197-202, Sendai, Japan, 1990.

[2] J. N. Yang and T. T. Soong, "Recent advances in active control of civil engineering structures," Probabilistic Engineering Mechanics, vol. 3, no. 4, pp. 179-188, 1988.

[3] R. K. Miller, S. F. Masri, T. J. Dehghanyar, and T. K. Caughey, "Active vibration control of large civil structures," Journal of Engineering Mechanics, vol. 114, no. 9, pp. 1542-1570, 1988.

[4] A. M. Reinhorn and G. D. Manolis, "Recent advances in structural control," Shock \& Vibration Digest, vol. 21, no. 1, pp. 3-8, 1989.

[5] A. E. Bryson Jr., "Optimal control-1950 to 1985," IEEE Control Systems, vol. 16, no. 3, pp. 26-33, 1996.

[6] H. J. Sussmann and J. C. Willems, "300 years of optimal control: from the brachystochrone to the maximum principle," IEEE Control Systems Magazine, vol. 17, no. 3, pp. 32-44, 1997.

[7] A. Jajarmi, N. Pariz, A. Vahidian Kamyad, and S. Effati, "A highly computational efficient method to solve nonlinear optimal control problems," Scientia Iranica, vol. 19, no. 3, pp. 759-766, 2012.

[8] R. V. Grandhi, "Structural and control optimization of space structures," Computers \& Structures, vol. 31, no. 2, pp. 139-150, 1989.

[9] J. M. Sloss, J. C. Bruch Jr., and I. S. Sadek, "A maximum principle for nonconservative self-adjoint systems," IMA Journal of Mathematical Control and Information, vol. 6, no. 2, pp. 199-216, 1989.

[10] S. Adali, J. C. Brunch Jr., I. S. Sadek, and J. M. Sloss, "Simultaneous design/control optimization of symmetric cross-ply laminates hybrid construction," Engineering Optimization, vol. 17, pp. 123-140, 1991.

[11] S. Adali, M. Walker, and V. E. Verijenko, "Multiobjective optimization of laminated plates for maximum prebuckling, buckling and postbuckling strength using continuous and discrete ply angles," Composite Structures, vol. 35, no. 1, pp. 117-130, 1996.

[12] J. M. Sloss, I. S. Sadek, J. C. Bruch Jr., and S. Adali, "Design/control optimization of cross-ply laminates under buckling and vibration," Journal of Aerospace Engineering, vol. 5, no. 1, pp.127137, 1992. 
[13] I. S. Sadek, J. M. Sloss, S. Adali, and J. C. Bruch Jr., "Integrated design and control of laminated hybrid plates with dynamic response and buckling objectives," Journal of Sound and Vibration, vol. 163, no. 1, pp. 57-66, 1993.

[14] M. E. Fares, Y. G. Youssif, and A. E. Alamir, "Optimal design and control of composite laminated plates with various boundary conditions using various plate theories," Composite Structures, vol. 56, no. 1, pp. 1-12, 2002.

[15] M. E. Fares, Y. G. Youssif, and M. A. Hafiz, "Structural and control optimization for maximum thermal buckling and minimum dynamic response of composite laminated plates," International Journal of Solids and Structures, vol. 41, no. 3-4, pp. 1005-1019, 2004.

[16] M. E. Fares, Y. G. Youssif, and M. A. Hafiz, "Optimal control of the dynamic response of an anisotropic plate with various boundary conditions," Mechanics Research Communications, vol. 28, no. 5, pp. 525-534, 2001.

[17] M. E. Fares, Y. G. Youssif, and A. E. Elshoraky, "Non-linear design and control optimization of composite laminated plates with buckling and postbuckling objectives," International Journal of Non-Linear Mechanics, vol. 41, no. 6-7, pp. 807-824, 2006.

[18] P. Dash and B. N. Singh, "Buckling and post-buckling of laminated composite plates," Mechanics Research Communications, vol. 46, pp. 1-7, 2012.

[19] A. Lal, K. R. Jagtap, and B. N. Singh, "Post buckling response of functionally graded materials plate subjected to mechanical and thermal loadings with random material properties," Applied Mathematical Modelling, vol. 37, no. 5, pp. 2900-2920, 2013.

[20] M. E. Fares, M. K. Elmarghany, and D. Atta, "An efficient and simple refined theory for bending and vibration of functionally graded plates," Composite Structures, vol. 91, no. 3, pp. 296-305, 2009.

[21] R. Bellman, Dynamic Programming, Princeton University Press, 1957.

[22] T. Mori and K. Tanaka, "Average stress in matrix and average elastic energy of materials with misfitting inclusions," Acta Metallurgica, vol. 21, no. 5, pp. 571-574, 1973. 

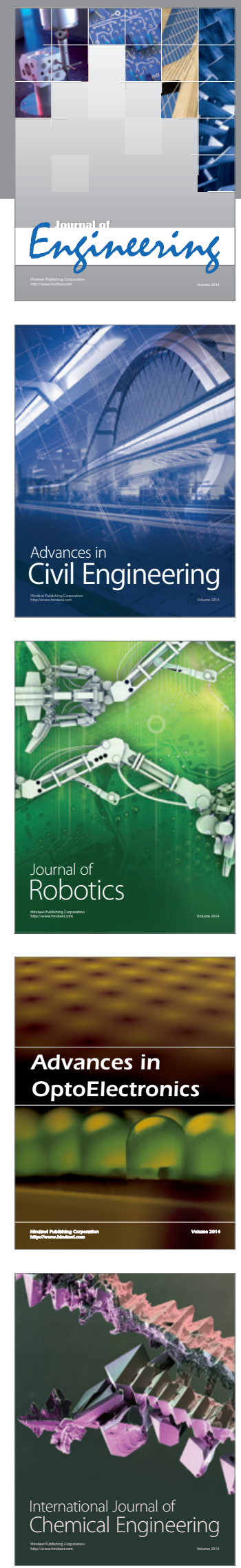

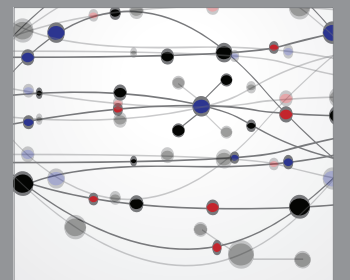

The Scientific World Journal
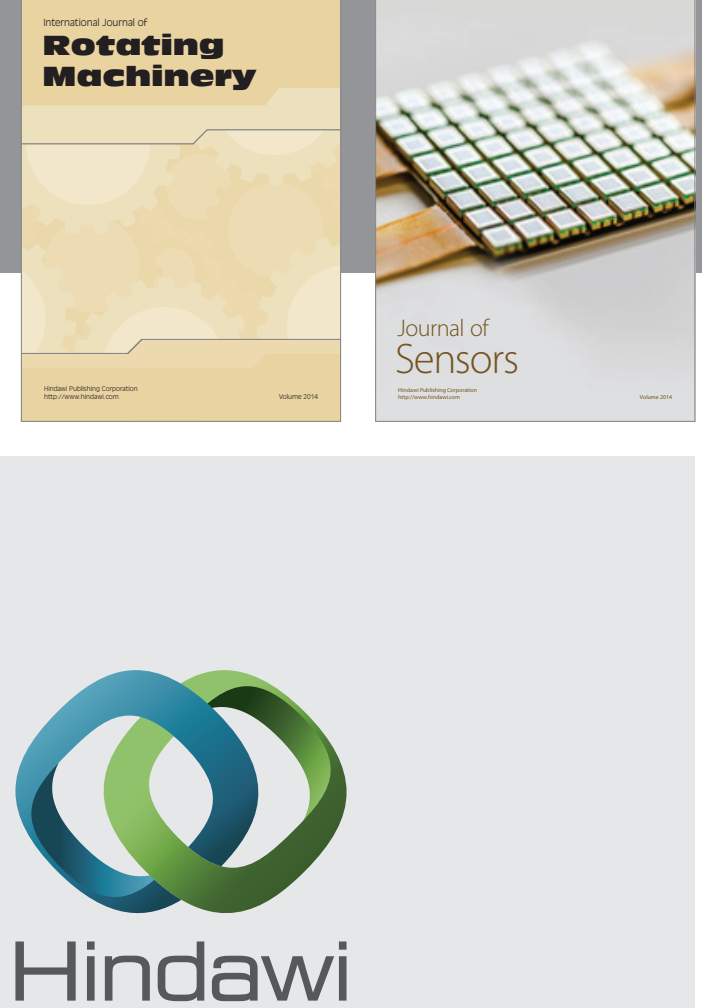

Submit your manuscripts at http://www.hindawi.com
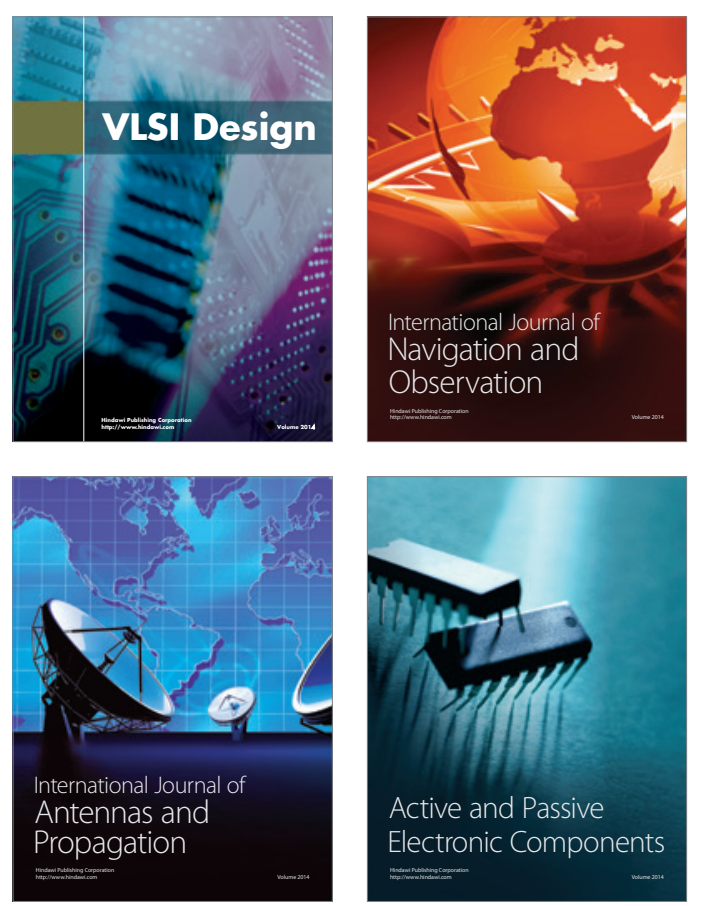
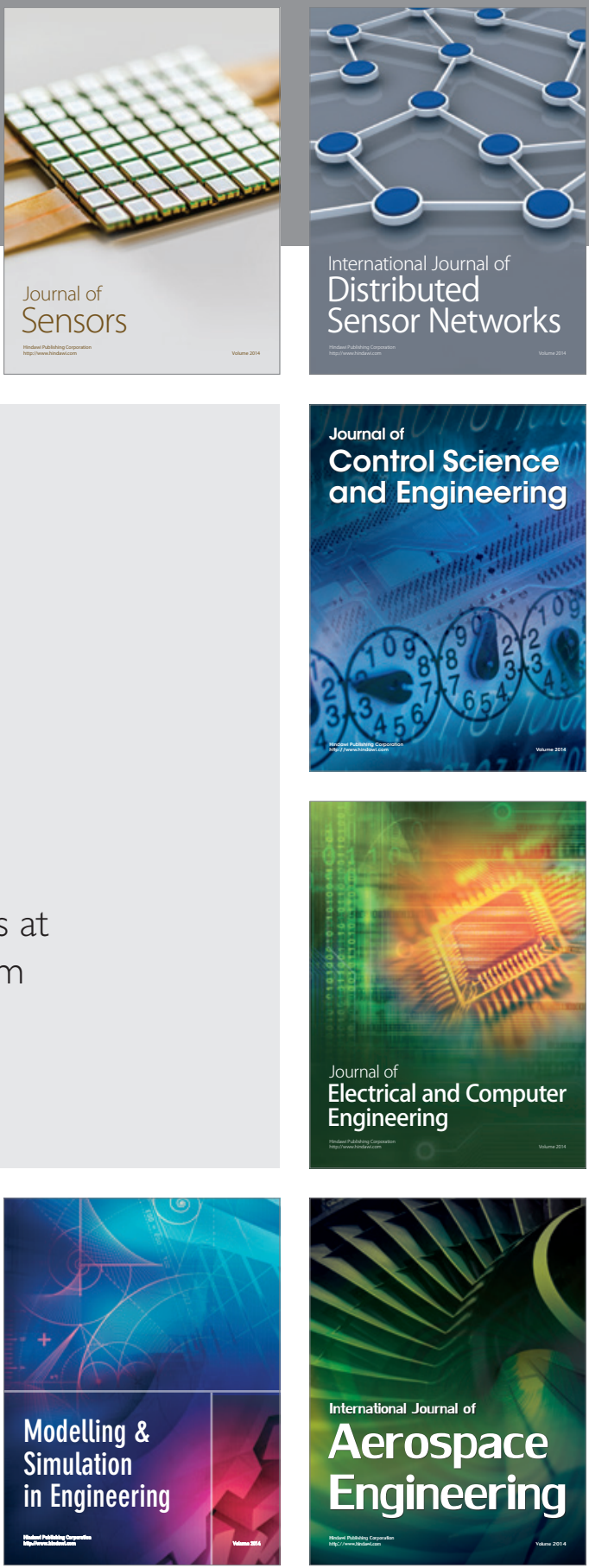

Journal of

Control Science

and Engineering
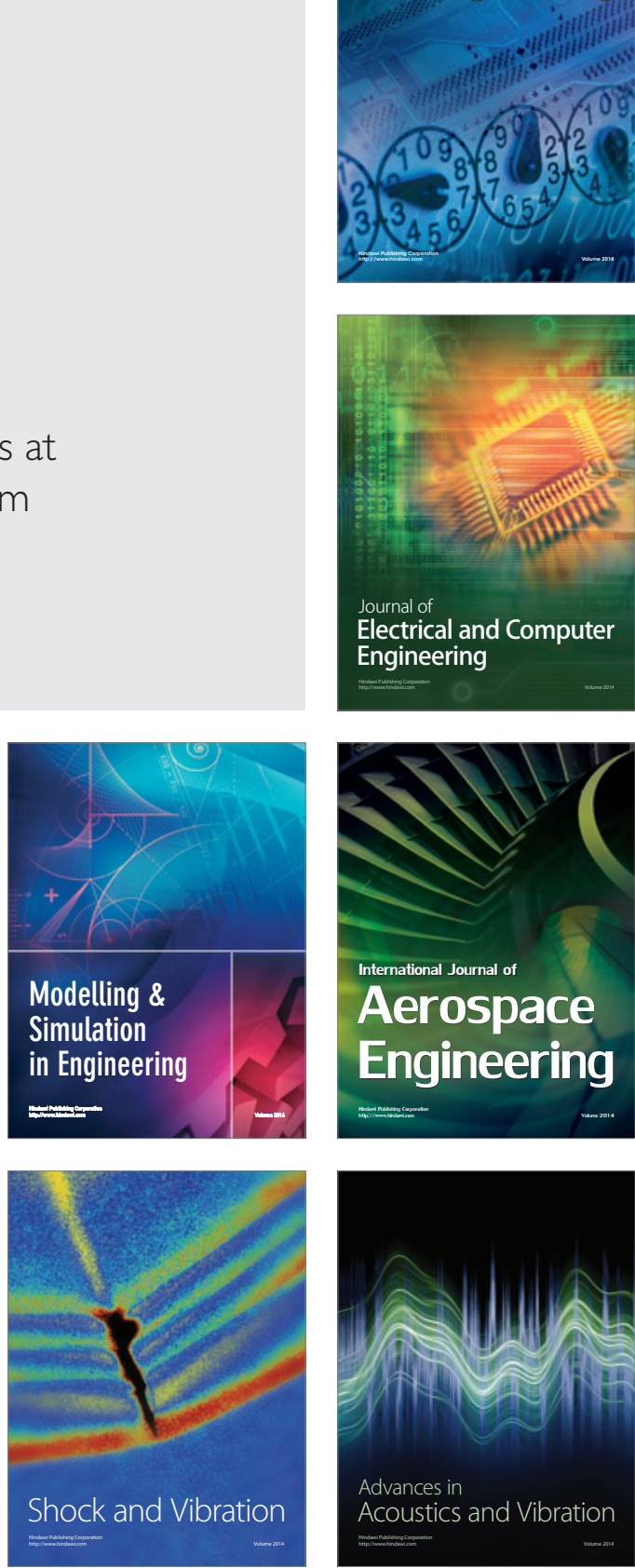